\title{
Article
}

\section{Design of Static Output Feedback and Structured Controllers for Active Suspension with Quarter-Car Model}

\author{
Manbok Park ${ }^{1}\left[\right.$ and Seongjin Yim ${ }^{2, *}$ (D) \\ 1 Department of Electronic Engineering, Korea National University of Transportation, Chungju-si 27469, Korea; \\ ohnnuri@ut.ac.kr \\ 2 Research Center for Electrical and Information Technology, Seoul National University of Science \\ and Technology, Seoul 01811, Korea \\ * Correspondence: acebtif@seoultech.ac.kr; Tel.: +82-2-970-9011
}

Citation: Park, M.; Yim, S. Design of Static Output Feedback and Structured Controllers for Active Suspension with Quarter-Car Model. Energies 2021, 14, 8231. https:// doi.org/10.3390/en14248231

Academic Editor: Daniel Chindamo

Received: 16 November 2021

Accepted: 6 December 2021

Published: 7 December 2021

Publisher's Note: MDPI stays neutral with regard to jurisdictional claims in published maps and institutional affiliations.

Copyright: (c) 2021 by the authors. Licensee MDPI, Basel, Switzerland. This article is an open access article distributed under the terms and conditions of the Creative Commons Attribution (CC BY) license (https:// creativecommons.org/licenses/by/ $4.0 /)$.

\begin{abstract}
This paper presents a method to design active suspension controllers for a 7-Degree-ofFreedom (DOF) full-car (FC) model from controllers designed with a 2-DOF quarter-car (QC) one. A linear quadratic regulator (LQR) with 7-DOF FC model has been widely used for active suspension control. However, it is too hard to implement the LQR in real vehicles because it requires so many state variables to be precisely measured and has so many elements to be implemented in the gain matrix of the LQR. To cope with the problem, a 2-DOF QC model describing vertical motions of sprung and unsprung masses is adopted for controller design. LQR designed with the QC model has a simpler structure and much smaller number of gain elements than that designed with the FC one. In this paper, several controllers for the FC model are derived from LQR designed with the QC model. These controllers can give equivalent or better performance than that designed with the FC model in terms of ride comfort. In order to use available sensor signals instead of using full-state feedback for active suspension control, LQ static output feedback (SOF) and linear quadratic Gaussian (LQG) controllers are designed with the QC model. From these controllers, observer-based controllers for the FC model are also derived. To verify the performance of the controllers for the FC model derived from LQR and LQ SOF ones designed with the QC model, frequency domain analysis is undertaken. From the analysis, it is confirmed that the controllers for the FC model derived from LQ and LQ SOF ones designed with the QC model can give equivalent performance to those designed with the FC one in terms of ride comfort.
\end{abstract}

Keywords: active suspension control; 2-DOF quarter-car model; 7-DOF full-car model; linear quadratic regulator; LQ static output feedback control; LQG

\section{Introduction}

The main objective in designing suspension is to improve ride comfort and road adhesion. Ride comfort can be evaluated with the vertical acceleration of a sprung mass of a vehicle. Figure 1 shows the frequency weightings representing the sensitivity of the human body to vibration, specified in ISO2631-1 [1]. According to Figure 1, human bodies are considered to be most sensitive to frequencies in the $0.5-2 \mathrm{~Hz}$ range in horizontal direction and in the $4-10 \mathrm{~Hz}$ range in vertical direction. So, it is necessary to reduce the vertical acceleration of the sprung mass near $4-10 \mathrm{~Hz}$ range for the ride comfort [2]. Road adhesion can be evaluated with suspension stroke and tire deflection. Generally, it has been known that there is trade-off between the ride comfort and road adhesion [3]. 


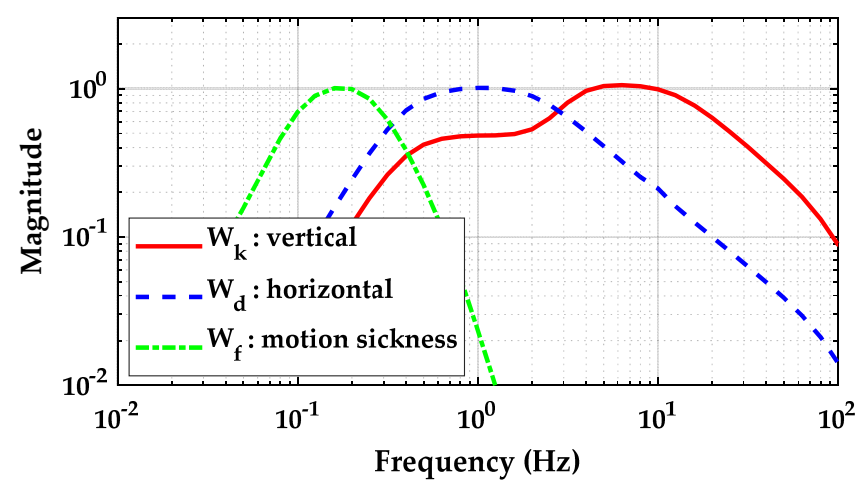

Figure 1. Frequency weightings specified in ISO 2631-1 standard [2].

There have been several devices for controlling the suspension in vehicles, such as active suspension, continuous damping control and air spring, etc. Among them, active suspension has been known to be the most effective for enhancing ride comfort and road adhesion. As a result, there have been a lot of studies on active suspension control. Comprehensive surveys on active suspension control can also be found in [3-7]. Recent advances for the last decade in active suspension control are summarized in [8].

Several suspension models have been used in designing a controller for the active suspension. Typical models are 2-Degree-of-Freedom (DOF) quarter-car (QC), 4-DOF half-car (HC) and 7-DOF full-car (FC) ones [6]. Nonlinear elements or actuators have been included in suspension models $[9,10]$. A typical example including nonlinear elements and actuators is an in-wheel motor system, which has been developed for the last decade [11-13]. To capture nonlinearities in suspension models, a Takagi-Sugeno (T-S) or interval fuzzy model has been adopted [14-16]. Among these models, 7-DOF linear FC model have been preferred because it can represent the vertical, roll and pitch motions of a sprung mass or vehicle body [3]. However, the FC model is so complex that it is not easy to implement a controller for active suspension.

Several methodologies such as linear optimal control, $H_{\infty}$ control, nonlinear control, and adaptive control theories have been applied in designing an active suspension controller [3-8]. Among these methodologies, linear quadratic regulator (LQR) has been widely adopted with 2-DOF QC and 7-DOF FC models because it can provide a systematic way to design a controller for active suspension. $\mathrm{LQR}$ is a full-state feedback controller, which requires all state variables to be measured. However, some of state variables of 7-DOF FC model cannot be measured in real vehicles. For example, the vertical displacement, the roll and pitch angles of a sprung mass are too hard to be measured with commercial sensors. For this reason, a state observer has been adopted for the purpose of estimating state variables of the FC model. Generally, the Kalman filter has been widely adopted as a state observer for LQR. With the Kalman filter and LQR, the linear quadratic Gaussian (LQG) for active suspension control has been extensively studied [17-19].

Another drawback in using LQR or LQG with the FC model for active suspension control is that there are so many state variables and control inputs. For example, the FC model has 14 state variables and four control inputs. If $L Q R$ is adopted for active suspension control, the dimension of gain matrix is $4 \times 14$. As a result, it is not easy to implement LQR or LQG on real vehicles. Therefore, it is necessary to design a simple controller, which requires a smaller number of state variables and is easy to implement in real vehicles.

In this paper, a 2-DOF QC model describing the vertical motions of sprung and unsprung masses is used to design a controller for active suspension. LQR is adopted to design the controller with the QC model. Let this controller be denoted as LQRq. LQRq has four elements in the gain matrix. Let the LQR designed with the FC model be denoted as LQRf. LQRf has 56 elements in the gain matrix. Based on the fact that the FC model consists of four QC ones, a full-state feedback controller for the FC model can derived from 
LQRq with some matrix manipulations. Let this controller be denoted as LQRfq. This is the key contribution of this paper. In LQRfq, the number of distinct gain elements is four, which is borrowed from LQRq. So, LQRfq is much simpler than LQRf and requires a much smaller number of gain elements in the gain matrix. LQRfq can give an equivalent or better performance than LQRf in terms of the ride comfort. This idea was presented in the previous work [20]. In the work, a proportional-integral-derivative (PID) control was adopted with the suspension stroke signal. With this method, it is not easy to evaluate and validate performances of the designed controller. In view of the controller for the FC model, LQRfq is a static output feedback (SOF) or structured controller. Therefore, this controller can be directly designed using LQ SOF control methodology.

Although the number of state variables needed for LQRq is smaller than those for LQRf, it is still not easy to measure some state variables such as vertical displacement of the sprung mass. To cope with the problem, a LQ SOF controller is designed with the QC model and available sensor signals [21,22]. Let this controller be denoted as LQSOFq. Following the identical procedure from LQRq to LQRfq, a full-state feedback controller for the FC model is derived from LQSOFq, designed with the QC model. Let this controller be denoted as LQSOFfq. The number of gain elements needed for LQSOFfq is just 2, which is borrowed from LQSOFq. Moreover, LQSOFfq can give equivalent or better performance than LQRf in terms of the ride comfort.

Another method to cope with the problem is to use an observer-based output feedback controller. LQG is the typical example. In this paper, LQG is designed with the QC and FC models. Let the LQG designed with the QC and FC models be denoted as LQGq and LQGf, respectively. When designing an observer-based output feedback controller for the FC model, the designed controllers from the QC models, i.e., LQRfq and LQSOFfq, are adopted as a full-state feedback controller.

To verify the performance of the controllers derived from ones designed with the QC model, frequency-domain analysis with singular value plot is conducted for the FC model. The full-state feedback, static-output feedback and observer-based output feedback controllers are compared in terms of the ride comfort.

This paper is organized as follows: In Section 2, 2-DOF QC and 7-DOF FC models are presented and modeled as a state-space equation. Based on the QC model, the controller for active suspension is design with LQR. SOF and LQG controllers are also designed with the $\mathrm{QC}$ and FC models. In Section 3, frequency-domain analysis with singular value plot is done. The conclusions are given in Section 4.

\section{Controller Design for Active Suspension}

In this section, the state-space equations for 2-DOF quarter-car and 7-DOF full-car modes following the procedure presented in the previous research $[23,24]$. In these studies, vector-matrix forms of equations of motions were derived and merged into a single statespace equation. Hereafter, the subscripts $q$ and $f$ mean controllers designed with the QC and FC models, respectively. The subscript $f q$ means a controller for the FC model derived from that designed with the QC model.

\subsection{Controller Design with Quarter-Car Model}

Figure 2 shows a 2-DOF QC model, which describes the vertical motions of sprung and unsprung masses. As shown in Figure 2, the control input $u$ generated by an active suspension is located between the sprung and unsprung masses, $m_{s}$ and $m_{u}$. The disturbance is the road profile, $z_{r}$. 


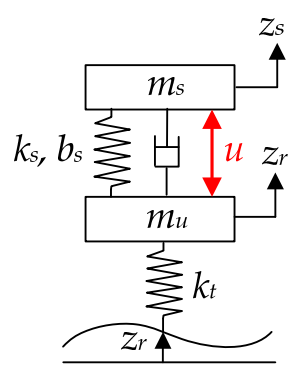

Figure 2. 2-DOF quarter-car model.

The force acting on suspension is derived as (1). The equations of vertical motions of the sprung and unsprung masses are given in (2). By combining (1) and (2), and changing it into vector-matrix form, (3) is obtained.

$$
\begin{gathered}
f=-k_{s}\left(z_{s}-z_{u}\right)-b_{s}\left(\dot{z}_{s}-\dot{z}_{u}\right)+u \\
\left\{\begin{array}{l}
m_{s} \ddot{z}_{s}=f \\
m_{u} \ddot{z}_{u}=-f-k_{t}\left(z_{u}-z_{r}\right)
\end{array}\right.
\end{gathered}
$$

$\left[\begin{array}{cc}m_{s} & 0 \\ 0 & m_{u}\end{array}\right]\left[\begin{array}{l}\ddot{z}_{s} \\ \ddot{z}_{u}\end{array}\right]=\left[\begin{array}{cc}-k_{s} & k_{s} \\ k_{s} & -k_{s}-k_{t}\end{array}\right]\left[\begin{array}{c}z_{s} \\ z_{u}\end{array}\right]+\left[\begin{array}{cc}-b_{s} & b_{s} \\ b_{s} & -b_{s}\end{array}\right]\left[\begin{array}{c}\dot{z}_{s} \\ \dot{z}_{u}\end{array}\right]+\left[\begin{array}{c}1 \\ -1\end{array}\right] u+\left[\begin{array}{c}0 \\ k_{t}\end{array}\right] z_{r}$

New vector and matrices are defined as (4) and (5), respectively $[23,24]$. With those definitions, (3) is rewritten as (6). The state vector of the QC model is defined as (7). With the definitions of new matrices as given in (8), the state-space equation for QC model is obtained as (9).

$$
\begin{aligned}
& \mathbf{z}=\left[\begin{array}{ll}
z_{s} & z_{u}
\end{array}\right]^{T} \\
& \mathbf{M}_{q} \triangleq\left[\begin{array}{cc}
m_{s} & 0 \\
0 & m_{u}
\end{array}\right], \mathbf{K}_{q} \triangleq\left[\begin{array}{cc}
-k_{s} & k_{s} \\
k_{s} & -k_{s}-k_{t}
\end{array}\right], \mathbf{B}_{q} \triangleq\left[\begin{array}{cc}
-b_{s} & b_{s} \\
b_{s} & -b_{s}
\end{array}\right], \mathbf{U}_{q} \triangleq\left[\begin{array}{c}
1 \\
-1
\end{array}\right], \mathbf{L}_{q} \triangleq\left[\begin{array}{c}
0 \\
k_{t}
\end{array}\right] \\
& \mathbf{M}_{q} \ddot{\mathbf{z}}=\mathbf{K}_{q} \mathbf{z}+\mathbf{B}_{q} \dot{\mathbf{z}}+\mathbf{U}_{q} u+\mathbf{L}_{q} z_{r} \\
& \mathbf{x}_{q}=\left[\begin{array}{c}
\mathbf{z} \\
\dot{\mathbf{z}}
\end{array}\right]=\left[\begin{array}{llll}
z_{s} & z_{u} & \dot{z}_{s} & \dot{z}_{u}
\end{array}\right]^{T} \\
& \mathbf{A}_{q} \triangleq\left[\begin{array}{cc}
0_{2 \times 2} & \mathbf{I}_{2 \times 2} \\
\mathbf{M}_{q}^{-1} \mathbf{K}_{q} & \mathbf{M}_{q}^{-1} \mathbf{B}_{q}
\end{array}\right], \mathbf{B}_{1 q} \triangleq\left[\begin{array}{c}
0_{2 \times 1} \\
\mathbf{M}_{q}^{-1} \mathbf{L}_{q}
\end{array}\right], \mathbf{B}_{2 q} \triangleq\left[\begin{array}{c}
0_{2 \times 1} \\
\mathbf{M}_{q}^{-1} \mathbf{U}_{q}
\end{array}\right] \\
& \dot{\mathbf{x}}_{q}=\mathbf{A}_{q} \mathbf{x}_{q}+\mathbf{B}_{1 q} z_{r}+\mathbf{B}_{2 q} u
\end{aligned}
$$

The LQ objective function for active suspension control is given as (10). The weights $\rho_{i}$ can be set to the inverse of the square of the maximum allowable value by Bryson's rule, i.e., $\rho_{i}=1 / \eta_{i}^{2}$ [25]. For ride comfort, the weight $\rho_{1}$ on the vertical acceleration of the sprung mass should be set to higher values while maintaining the other weights constant. On the other hand, for road adhesion or cornering, the weight $\rho_{3}$ on the tire deflection should be set to higher. Generally, these two objectives, ride comfort and road adhesion, conflict with each other. The regulated output $\mathbf{z}_{q}$ is defined as (11) with the state vector $\mathbf{x}_{q}$ and the control input $u$. With (11), the weighting matrices in $J_{q}$ are obtained as (12). With (12), the LQ objective function (10) can be rewritten into the vector-matrix form, (13). LQR is a controller with the form of full-state feedback, $\boldsymbol{u}=-\mathbf{K}_{q} \mathbf{x}_{q}$, which minimizes $J_{q}$. It is easy to obtain $\mathbf{K}_{q}$ from Riccati equation for a given set of weights, i.e., the matrices $\mathbf{Q}_{q}, \mathbf{N}_{q}$ and $\mathbf{R}_{q}$, as given in (12). Let denote this controller $\mathbf{K}_{q}$ as LQRq.

$$
J_{q}=\int_{0}^{\infty}\left\{\rho_{1} \ddot{z}_{s}^{2}+\rho_{2}\left(z_{s}-z_{u}\right)^{2}+\rho_{3} z_{u}^{2}+\rho_{4} u^{2}\right\} d t
$$




$$
\begin{gathered}
\mathbf{z}_{q}=\mathbf{C}_{q} \mathbf{x}_{q}+\mathbf{D}_{q} u \\
\mathbf{Q}_{q}=\mathbf{C}_{q}^{T} \mathbf{C}_{q}, \mathbf{N}_{q}=\mathbf{C}_{q}^{T} \mathbf{D}_{q}, \mathbf{R}_{q}=\mathbf{D}_{q}^{T} \mathbf{D}_{q} \\
J_{q}=\int_{0}^{\infty} \mathbf{z}_{q}^{T} \mathbf{z}_{q} d t=\int_{0}^{\infty}\left\{\left[\begin{array}{c}
\mathbf{x}_{q} \\
u
\end{array}\right]^{T}\left[\begin{array}{cc}
\mathbf{Q}_{q} & \mathbf{N}_{q} \\
\mathbf{N}_{q}^{T} & \mathbf{R}_{q}
\end{array}\right]\left[\begin{array}{c}
\mathbf{x}_{q} \\
u
\end{array}\right]\right\} d t
\end{gathered}
$$

\subsection{Controller Design with Full-Car Model}

Figure 3 shows a 7-DOF FC model, which describes the vertical, roll and pitch motions of a sprung mass, and the vertical motions of four unsprung masses. As shown in Figure 3, the indices of a suspension are in order of front left, front right, rear left and rear right ones, represented as (1), (2), (3) and (4), respectively. In the FC model, there are four external disturbances, i.e., the road profiles, $z_{r 1}, z_{r 2}, z_{r 3}$ and $z_{r 4}$, on the unsprung mass. Actuators located between the sprung and unsprung masses can generate the control inputs, $u_{1}, u_{2}$, $u_{3}$ and $u_{4}$, at each suspension.

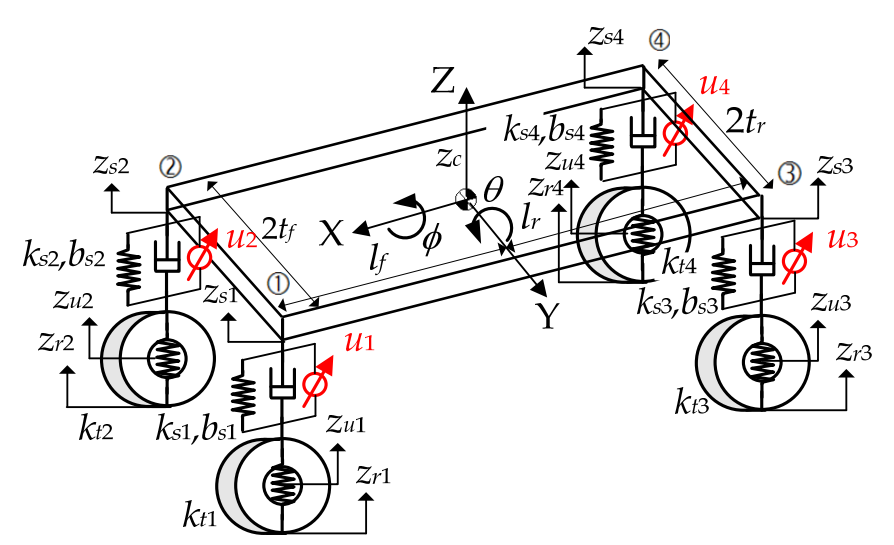

Figure 3. 7-DOF full-car model.

The suspension forces in the FC model are derived as (14). In (14), $u_{i}$ is the control input or force acting on the $i$-th suspension, which is identical to (1). The equations of motion for the sprung and unsprung masses are given in (15) and (16), respectively.

$$
\begin{aligned}
f_{i}=- & k_{s i}\left(z_{s i}-z_{u i}\right)-b_{s i}\left(\dot{z}_{s i}-\dot{z}_{u i}\right)+u_{i}, i=1 \cdots 4 \\
& \left\{\begin{array}{l}
m_{s} \ddot{z}_{c}=f_{1}+f_{2}+f_{3}+f_{4} \\
I_{x} \ddot{\phi}=t_{f} \cdot f_{1}-t_{f} \cdot f_{2}+t_{r} \cdot f_{3}-t_{r} \cdot f_{4} \\
I_{y} \ddot{\theta}=-l_{f} \cdot\left(f_{1}+f_{2}\right)+l_{r} \cdot\left(f_{3}+f_{4}\right)
\end{array}\right. \\
& \left\{\begin{array}{l}
m_{u 1} \ddot{z}_{u 1}=-f_{1}+k_{t 1}\left(z_{u 1}-z_{r 1}\right) \\
m_{u 2} \ddot{z}_{u 2}=-f_{2}+k_{t 2}\left(z_{u 2}-z_{r 2}\right) \\
m_{u 3} \ddot{z}_{u 3}=-f_{3}+k_{t 3}\left(z_{u 3}-z_{r 3}\right) \\
m_{u 4} \ddot{z}_{u 4}=-f_{4}+k_{t 4}\left(z_{u 4}-z_{r 4}\right)
\end{array}\right.
\end{aligned}
$$

In (14), the vertical displacement of each corner, $z_{s i}$, is not a state variable. Therefore, it should be derived from state variables. Figure 4 shows the roll and pitch motions of the sprung mass. As shown in Figure 4, the vertical displacements of the sprung mass, i.e., $z_{s 1}, z_{s 2}, z_{s 3}$ and $z_{s 4}$, at each corner are derived as (17) from the geometrical relationship under the assumption that the sprung mass is a rigid body. As shown in (17), there is a sine function, which is nonlinear. Generally, the roll and pitch angles are less than $5 \mathrm{deg}$ for most cases of vehicle driving conditions. Under the condition that the roll and pitch angles are small or less than $15 \mathrm{deg}$, i.e., $\sin \theta \simeq \theta$ and $\sin \phi \simeq \phi$, (17) can be rewritten as the vector-matrix form of (18) [23,24]. In (18), the matrix $G$ represents the geometric 
relationship between the vertical motions of four corners and the vertical, roll and pitch ones of the sprung mass.

$$
\begin{gathered}
\left\{\begin{array}{l}
z_{s 1}=z_{c}+t_{f} \cdot \sin \phi-l_{f} \cdot \sin \theta \\
z_{s 2}=z_{c}-t_{f} \cdot \sin \phi-l_{f} \cdot \sin \theta \\
z_{s 3}=z_{c}+t_{f} \cdot \sin \phi+l_{r} \cdot \sin \theta \\
z_{s 4}=z_{c}-t_{f} \cdot \sin \phi+l_{r} \cdot \sin \theta
\end{array}\right. \\
{\left[\begin{array}{l}
z_{s 1} \\
z_{s 2} \\
z_{s 3} \\
z_{s 4}
\end{array}\right]=\underbrace{\left[\begin{array}{ccc}
1 & t_{f} & -l_{f} \\
1 & -t_{f} & -l_{f} \\
1 & t_{r} & l_{r} \\
1 & -t_{r} & l_{r}
\end{array}\right]}_{\mathbf{G}^{T}}\left[\begin{array}{c}
z_{c} \\
\phi \\
\theta
\end{array}\right]=\mathbf{G}^{T}\left[\begin{array}{c}
z_{c} \\
\phi \\
\theta
\end{array}\right]}
\end{gathered}
$$

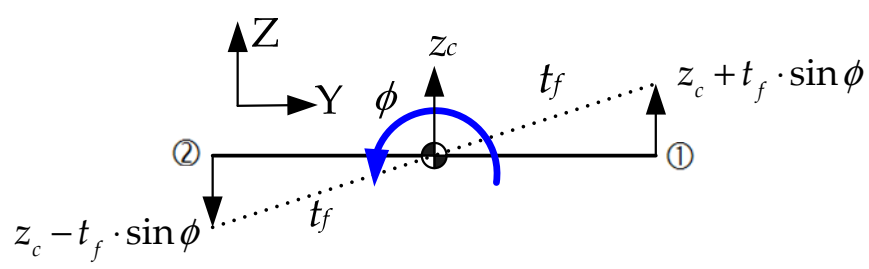

(a)

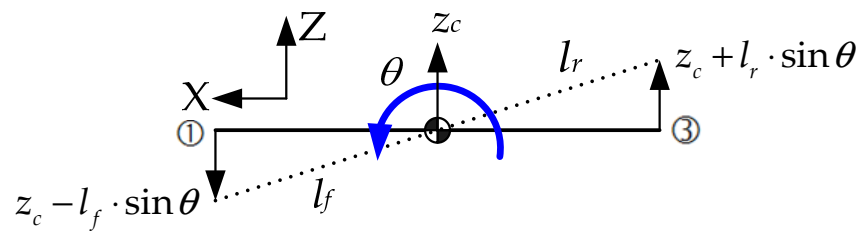

(b)

Figure 4. Roll and pitch motions of the sprung mass. (a) Roll motion (front view); (b) Pitch motion (side view).

For further derivation, the vectors of dynamic variables and the matrices of parameters are defined as (19) and (20), respectively $[23,24]$. In (20), $\operatorname{diag}(a, b, c, \ldots)$ represents the diagonal matrix consisting of the elements, $a, b, c, \ldots$ With those definitions, the Equations (15) and (16) are represented as the vector-matrix forms, (21). Moreover, the Equations (18) and (14) are represented as the vector-matrix forms, (22) and (23), respectively.

$$
\begin{aligned}
& \left\{\begin{array}{l}
\mathbf{z}_{s} \triangleq\left[\begin{array}{llll}
z_{s 1} & z_{s 2} & z_{s 3} & z_{s 4}
\end{array}\right]^{T}, \mathbf{z}_{u} \triangleq\left[\begin{array}{llll}
z_{u 1} & z_{u 2} & z_{u 3} & z_{u 4}
\end{array}\right]^{T}, \mathbf{z}_{r} \triangleq\left[\begin{array}{llll}
z_{r 1} & z_{r 2} & z_{r 3} & z_{r 4}
\end{array}\right]^{T} \\
\mathbf{p} \triangleq\left[\begin{array}{lll}
z_{c} & \phi & \theta
\end{array}\right]^{T}, \mathbf{u}_{f} \triangleq\left[\begin{array}{lllll}
u_{1} & u_{2} & u_{3} & u_{4}
\end{array}\right]^{T}, \mathbf{f} \triangleq\left[\begin{array}{llll}
f_{1} & f_{2} & f_{3} & f_{4}
\end{array}\right]^{T}
\end{array}\right. \\
& \left\{\begin{array}{l}
\mathbf{M}_{s} \triangleq \operatorname{diag}\left(m_{s}, I_{x}, I_{y}\right), \mathbf{M}_{u} \triangleq \operatorname{diag}\left(m_{u 1}, m_{u 2}, m_{u 3}, m_{u 4}\right) \\
\mathbf{K}_{s} \triangleq \operatorname{diag}\left(k_{s 1}, k_{s 2}, k_{s 3}, k_{s 4}\right), \mathbf{K}_{t} \triangleq \operatorname{diag}\left(k_{t 1}, k_{t 2}, k_{t 3}, k_{t 4}\right) \\
\mathbf{B}_{s} \triangleq \operatorname{diag}\left(b_{s 1}, b_{s 2}, b_{s 3}, b_{s 4}\right)
\end{array}\right. \\
& \left\{\begin{array}{l}
\mathbf{M}_{s} \ddot{\mathbf{p}}=\mathbf{G f} \\
\mathbf{M}_{u} \ddot{\mathbf{z}}_{u}=-\mathbf{f}+\mathbf{K}_{t}\left(\mathbf{z}_{u}-\mathbf{z}_{r}\right)
\end{array}\right. \\
& \mathbf{z}_{S}=\mathbf{G}^{T} \mathbf{p} \\
& \mathbf{f}=-\mathbf{K}_{s}\left(\mathbf{z}_{s}-\mathbf{z}_{u}\right)-\mathbf{B}_{s}\left(\dot{\mathbf{z}}_{s}-\dot{\mathbf{z}}_{u}\right)+\mathbf{u}_{f}=-\mathbf{K}_{s}\left(\mathbf{G}^{T} \mathbf{p}-\mathbf{z}_{u}\right)-\mathbf{B}_{s}\left(\mathbf{G}^{T} \dot{\mathbf{p}}-\dot{\mathbf{z}}_{u}\right)+\mathbf{u}_{f}
\end{aligned}
$$


By replacing $\mathbf{f}$ of (21) with (23), (21) is converted into (24). (24) is rearranged into (25) as the vector-matrix form. The vectors and matrices in (25) are defined as (26) and (27), respectively. With those definitions, (25) is rewritten into (28).

$$
\begin{gathered}
\left\{\begin{array}{r}
\mathbf{M}_{s} \ddot{\mathbf{p}}=-\mathbf{G K}_{s}\left(\mathbf{G}^{T} \mathbf{p}-\mathbf{z}_{u}\right)-\mathbf{G B}_{s}\left(\mathbf{G}^{T} \dot{\mathbf{p}}-\dot{\mathbf{z}}_{u}\right)+\mathbf{G} \mathbf{u}_{f} \\
\mathbf{M}_{u} \ddot{\mathbf{z}}_{u}=\mathbf{K}_{s}\left(\mathbf{G}^{T} \mathbf{p}-\mathbf{z}_{u}\right)+\mathbf{B}_{s}\left(\mathbf{G}^{T} \dot{\mathbf{p}}-\dot{\mathbf{z}}_{u}\right)+\mathbf{K}_{t}\left(\mathbf{z}_{u}-\mathbf{z}_{r}\right)-\mathbf{u}_{f}
\end{array}\right. \\
{\left[\begin{array}{cc}
\mathbf{M}_{s} & 0 \\
0 & \mathbf{M}_{u}
\end{array}\right]\left[\begin{array}{c}
\ddot{\mathbf{p}} \\
\ddot{\mathbf{z}_{u}}
\end{array}\right]=\left[\begin{array}{cc}
-\mathbf{G K}_{s} \mathbf{G}^{T} & \mathbf{G} \mathbf{K}_{s} \\
\mathbf{K}_{s} \mathbf{G}^{T} & -\mathbf{K}_{s}+\mathbf{K}_{t}
\end{array}\right]\left[\begin{array}{c}
\mathbf{p} \\
\mathbf{z}_{u}
\end{array}\right]} \\
+\left[\begin{array}{cc}
-\mathbf{G B}_{s} \mathbf{G}^{T} & \mathbf{G} \mathbf{B}_{s} \\
\mathbf{B}_{s} \mathbf{G}^{T} & -\mathbf{B}_{s}
\end{array}\right]\left[\begin{array}{c}
\dot{\mathbf{p}} \\
\dot{\mathbf{z}}_{u}
\end{array}\right]+\left[\begin{array}{c}
\mathbf{G} \\
-\mathbf{I}
\end{array}\right] \mathbf{u}_{f}+\left[\begin{array}{c}
0 \\
-\mathbf{K}_{t}
\end{array}\right] \mathbf{z}_{r} \\
\left\{\begin{array}{c}
\mathbf{q} \triangleq\left[\begin{array}{c}
\mathbf{p} \\
\mathbf{z}_{u}
\end{array}\right], \mathbf{w}_{f} \triangleq \mathbf{z}_{r} \\
\mathbf{M}_{e} \triangleq\left[\begin{array}{cc}
\mathbf{M}_{s} & 0 \\
0 & \mathbf{M}_{u}
\end{array}\right], \mathbf{K}_{e} \triangleq\left[\begin{array}{c}
-\mathbf{G K}_{s} \mathbf{G}^{T} \\
\mathbf{K}_{s} \mathbf{G}^{T}-\mathbf{K}_{s}+\mathbf{K}_{t}
\end{array}\right] \\
\mathbf{B}_{e} \triangleq\left[\begin{array}{c}
-\mathbf{G B}_{s} \mathbf{G}^{T} \\
\mathbf{B}_{s} \mathbf{G}^{T} \\
\mathbf{G B}_{s} \\
-\mathbf{B}_{s}
\end{array}\right], \mathbf{U}_{e} \triangleq\left[\begin{array}{c}
\mathbf{G} \\
-\mathbf{I}
\end{array}\right], \mathbf{L}_{e} \triangleq\left[\begin{array}{c}
0 \\
-\mathbf{K}_{t}
\end{array}\right]
\end{array}\right.
\end{gathered}
$$

The state vector of the FC model is defined as (29). With the definitions of matrices as given in (30), the state-space equation of the 7-DOF FC model is obtained as (31).

$$
\begin{gathered}
\mathbf{x}_{f} \triangleq\left[\begin{array}{c}
\mathbf{q} \\
\dot{\mathbf{q}}
\end{array}\right] \\
\mathbf{A}_{f}=\left[\begin{array}{cc}
0 & \mathbf{I}_{7 \times 7} \\
\mathbf{M}_{e}^{-1} \mathbf{K}_{e} & \mathbf{M}_{e}^{-1} \mathbf{B}_{e}
\end{array}\right], \mathbf{B}_{1 f}=\left[\begin{array}{c}
0_{7 \times 4} \\
\mathbf{M}_{e}^{-1} \mathbf{L}_{e}
\end{array}\right], \mathbf{B}_{2 f}=\left[\begin{array}{c}
0_{7 \times 4} \\
\mathbf{M}_{e}^{-1} \mathbf{U}_{e}
\end{array}\right] \\
\dot{\mathbf{x}}_{f}=\mathbf{A}_{f} \mathbf{x}_{f}+\mathbf{B}_{1 f} \mathbf{w}_{f}+\mathbf{B}_{2 f} \mathbf{u}_{f}
\end{gathered}
$$

The LQ objective function for active suspension control with the FC model is given as (32). The weights $\zeta_{i}$ can be set to the inverse of the square of the maximum allowable value by Bryson's rule, i.e., $\zeta_{i}=1 / \xi_{i}^{2}$ [25]. For ride comfort, the weights $\xi_{1}, \xi_{2}$, and $\xi_{3}$ on the vertical, roll and pitch accelerations should be set to higher values. On the other hand, for road adhesion or cornering, the weights $\xi_{8}$ and $\xi_{9}$ on the suspension stroke and tire deflection should be set to higher. Generally, these two objectives, ride comfort and road adhesion, conflict with each other.

The regulated output $\mathbf{z}_{f}$ is defined as (33) with the state and input vectors, $\mathbf{x}_{f}$ and $\mathbf{u}_{f}$. With (33), the weighting matrices in $J_{f}$ are obtained as (34). With (34), the LQ objective function (32) can be rewritten into the vector-matrix form of (35). LQR is a controller with the form of full-state feedback, $\mathbf{u}_{f}=-\mathbf{K}_{f} \mathbf{x}_{f}$, which minimizes $J_{f}$. It is easy to obtain $\mathbf{K}_{f}$ from Riccati equation for a given set of weights, i.e., the weighting matrices $\mathbf{Q}_{f}, \mathbf{N}_{f}$ and $\mathbf{R}_{f}$, as given in (34). Let denote this controller $\mathbf{K}_{f}$ as LQRf1.

$$
\begin{gathered}
J_{f}=\int_{0}^{\infty}\left\{\begin{array}{c}
\xi_{1} \ddot{z}_{c}^{2}+\xi_{2} \ddot{\phi}^{2}+\xi_{3} \ddot{\theta}^{2}+\xi_{4} \phi^{2}+\xi_{5} \dot{\phi}^{2}+\xi_{6} \theta^{2} \\
+\xi_{7} \dot{\theta}^{2}+\xi_{8} \sum_{i=1}^{4}\left(z_{s i}-z_{u i}\right)^{2}+\xi_{9} \sum_{i=1}^{4} z_{u i}^{2}+\xi_{10} \sum_{i=1}^{4} u_{i}^{2}
\end{array}\right\} d t \\
\mathbf{z}_{f}=\mathbf{C}_{f} \mathbf{x}_{f}+\mathbf{D}_{f} \mathbf{u}_{f} \\
\mathbf{Q}_{f}=\mathbf{C}_{f}^{T} \mathbf{C}_{f}, \mathbf{N}_{f}=\mathbf{C}_{f}^{T} \mathbf{D}_{f}, \mathbf{R}_{f}=\mathbf{D}_{f}^{T} \mathbf{D}_{f}
\end{gathered}
$$




$$
J_{f}=\int_{0}^{\infty} \mathbf{z}_{f}^{T} \mathbf{z}_{f} d t=\int_{0}^{\infty}\left\{\left[\begin{array}{l}
\mathbf{x}_{f} \\
\mathbf{u}_{f}
\end{array}\right]^{T}\left[\begin{array}{ll}
\mathbf{Q}_{f} & \mathbf{N}_{f} \\
\mathbf{N}_{f}^{T} & \mathbf{R}_{f}
\end{array}\right]\left[\begin{array}{l}
\mathbf{x}_{f} \\
\mathbf{u}_{f}
\end{array}\right]\right\} d t
$$

The LQ objective function for the FC model, (32), can be converted into that for the QC one, (36), just like (10). This function, (36), has the identical form to (10). The weights $\sigma_{i}$ can be set to the inverse of the square of the maximum allowable value by Bryson's rule, i.e., $\sigma_{i}=1 / \chi_{i}^{2}$ [25]. With the new definitions of matrices as given in (37), the output and the weighting matrices are defined as (38) and (39), respectively. With these matrices, the LQ objective function (36) is represented as the vector-matrix form of (40). As mentioned earlier, it is easy to obtain $\mathbf{K}_{f}$ from the Riccati equation for a given set of weights, i.e., the matrices $\mathbf{Q}_{f q}, \mathbf{N}_{f q}$ and $\mathbf{R}_{f q}$, as given in (39). Let this controller be denoted as LQRf2. LQRf1 and LQRf2 are the full-state feedback controllers, which has different LQ objective functions, (32) and (36).

$$
\begin{gathered}
J_{f q}=\int_{0}^{\infty}\left\{\sigma_{1} \sum_{i=1}^{4} \ddot{z}_{s i}^{2}+\sigma_{2} \sum_{i=1}^{4}\left(z_{s i}-z_{u i}\right)^{2}+\sigma_{3} \sum_{i=1}^{4} z_{u i}^{2}+\sigma_{4} \sum_{i=1}^{4} u_{i}^{2}\right\} d t \\
\left\{\begin{array}{l}
\mathbf{C}_{z s a} \triangleq \mathbf{G}^{T}\left[\begin{array}{lll}
\mathbf{A}_{f, 8} & \mathbf{A}_{f, 9} & \mathbf{A}_{f, 10}
\end{array}\right]^{T} \\
\mathbf{C}_{z s b} \triangleq \mathbf{G}^{T}\left[\begin{array}{lll}
\mathbf{B}_{2 f, 8} & \mathbf{B}_{2 f, 9} & \mathbf{B}_{2 f, 10}
\end{array}\right]^{T} \\
\mathbf{C}_{s s q} \triangleq\left[\begin{array}{lll}
\mathbf{G}^{T} & -\mathbf{I}_{4 \times 4} & 0_{4 \times 7}
\end{array}\right] \\
\mathbf{C}_{z u q} \triangleq\left[\begin{array}{lll}
0_{3 \times 4} & \mathbf{I}_{4 \times 4} & 0_{4 \times 7}
\end{array}\right] \\
\mathbf{z}_{f q}=\mathbf{C}_{f q} \mathbf{x}_{f}+\mathbf{D}_{f q} \mathbf{u}_{f}=\left[\begin{array}{c}
\mathbf{C}_{z s a} \\
\mathbf{C}_{s s q} \\
\mathbf{C}_{z u q} \\
0_{4 \times 14}
\end{array}\right] \mathbf{x}_{f}+\left[\begin{array}{l}
\mathbf{C}_{z s b} \\
0_{4 \times 4} \\
0_{4 \times 7} \\
\mathbf{I}_{4 \times 4}
\end{array}\right] \mathbf{u}_{f} \\
\mathbf{Q}_{f q}=\mathbf{C}_{f q}^{T} \mathbf{C}_{f q}, \mathbf{N}_{f q}=\mathbf{C}_{f q}^{T} \mathbf{D}_{f q}, \mathbf{R}_{f q}=\mathbf{D}_{f q}^{T} \mathbf{D}_{f q} \\
J_{f q}=\int_{0}^{\infty} \mathbf{z}_{f q}^{T} \mathbf{z}_{f q} d t=\int_{0}^{\infty}\left\{\left[\begin{array}{c}
\mathbf{x}_{f} \\
\mathbf{u}_{f}
\end{array}\right]^{T}\left[\begin{array}{cc}
\mathbf{Q}_{f q} & \mathbf{N}_{f q} \\
\mathbf{N}_{f q}^{T} & \mathbf{R}_{f q}
\end{array}\right]\left[\begin{array}{l}
\mathbf{x}_{f} \\
\mathbf{u}_{f}
\end{array}\right]\right\} d t
\end{array}\right.
\end{gathered}
$$

LQR provides the systematic way to design the active suspension controller. Generally, LQR has the structure of full-state feedback. As shown in (29) and (19), i.e., $\mathbf{x}_{f}$ and $\mathbf{u}_{f}$, there are 14 state variables and 4 control inputs in the 7-DOF FC model, respectively. So, the dimension of the gain matrix $\mathbf{K}_{f}$ of $L Q R$ is $4 \times 14$. To implement the $L Q R$ in real vehicles, it is necessary to precisely measure or estimate 14 state variables. However, it is too hard to measure or estimate those variables in real vehicles. For this reason, it is necessary to design a controller which has the smaller number of state variables and can be used for the FC model.

\subsection{How to Use $L Q R$ for Quarter-Car Model as a Controller for Full-Car One}

As shown in Figures 2 and 3, it can be regarded that the FC model consists of four QC models. The vertical displacements of the sprung and unsprung masses, $z_{s i}$ and $z_{u i}$, in the FC model are identical to those of the QC one. Therefore, LQRq can be used to design a full-state feedback controller having the identical structure to LQRf. This subsection explains how to use LQRq in designing the controller.

Some of the state variables in the FC model can be grouped into those of the QC one, $\mathbf{x}_{q i}$, as given in (41). $\mathbf{K}_{q}$ in (42) represents the gain matrix of LQRq. By multiplying (42) and (41) together, the control input of active suspension at each corner is obtained as (43). With $\mathbf{K}_{q}$ in (42), the control input for FC model, $\mathbf{u}_{f}$, is calculated as (44), which has the identical form to (43). (44) is converted into (45) with the definition of (22). The four vectors in (45) can be represented by the state vector of the FC model, $\mathbf{x}_{f}$, as given in (46). With (46), the control input for the FC model is represented as (47) with the gain elements of $\mathbf{K}_{q}$. This 
means that the full-state feedback controller for the FC model can be derived from the gain elements of $\mathbf{K}_{q}$. As shown in (47), the number of gain elements needed to derive the full-state feedback controller for the FC model is just four, which is much smaller than that of $\mathbf{K}_{f}$. This is the key contribution of this paper.

As given in (48), the gain matrix $\mathbf{H}$ has a special structure. Therefore, it is different from the LQR gain matrix, $\mathbf{K}_{f}$. Let this controller $\mathbf{H}$ be denoted as LQRfq. It is expected that LQRfq can give an equivalent performance to LQRf2 because these adopt the identical LQ objective function, (10) and (36).

$$
\begin{aligned}
& \mathbf{x}_{q i}=\left[\begin{array}{llll}
z_{s i} & z_{u i} & \dot{z}_{s i} & \dot{z}_{u i}
\end{array}\right]^{T}, i=1,2,3,4 \\
& \mathbf{K}_{q}=\left[\begin{array}{llll}
k_{1} & k_{2} & k_{3} & k_{4}
\end{array}\right] \\
& u_{i}=-\mathbf{K}_{q} \mathbf{x}_{q i}=-\left(k_{1} z_{s i}+k_{2} z_{u i}+k_{3} \dot{z}_{s i}+k_{4} \dot{z}_{u i}\right), i=1,2,3,4 \\
& \mathbf{u}_{f}=k_{1} \mathbf{z}_{s}+k_{2} \mathbf{z}_{u}+k_{3} \dot{\mathbf{z}}_{s}+k_{4} \dot{\mathbf{z}}_{u} \\
& \mathbf{u}_{f}=k_{1} \mathbf{G}^{T} \mathbf{p}+k_{2} \mathbf{z}_{u}+k_{3} \mathbf{G}^{T} \dot{\mathbf{p}}+k_{4} \dot{\mathbf{z}}_{u}
\end{aligned}
$$

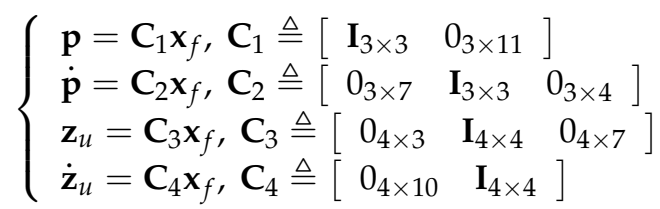

$$
\begin{aligned}
& \mathbf{u}_{f}=k_{1} \mathbf{G}^{T} \mathbf{C}_{1} \mathbf{x}_{f}+k_{2} \mathbf{C}_{3} \mathbf{x}_{f}+k_{3} \mathbf{G}^{T} \mathbf{C}_{2} \mathbf{x}_{f}+k_{4} \mathbf{C}_{4} \mathbf{x}_{f} \\
& =\left(k_{1} \mathbf{G}^{T} \mathbf{C}_{1}+k_{2} \mathbf{C}_{3}+k_{3} \mathbf{G}^{T} \mathbf{C}_{2}+k_{4} \mathbf{C}_{4}\right) \mathbf{x}_{f} \\
& =\mathbf{H} \mathbf{x}_{f} \\
& \mathbf{H}=\left[\begin{array}{cccccccc}
r_{1} & -r_{2} & r_{3} & & r_{6} & -r_{7} & r_{8} & \\
r_{1} & r_{2} & r_{3} & r_{5} \mathbf{I}_{4 \times 4} & r_{6} & r_{7} & r_{8} & r_{10} \mathbf{I}_{4 \times 4} \\
r_{1} & -r_{2} & r_{4} & & -r_{7} & r_{9} & \\
r_{1} & r_{2} & r_{4} & & r_{6} & r_{7} & r_{9} &
\end{array}\right]
\end{aligned}
$$

Indeed, the controller with the form of (47), i.e., LQRfq, is LQ static output feedback (SOF) or structured controller (SC). LQRfq is LQ SOF controller in that some of state variables, i.e., available sensor signals, are used for the feedback. It is also structured controller because $\mathbf{H}$ has a special structure, as given in (48). In (48), $r_{i}$ are distinct gain elements. All the controllers derived from (47) have the identical structure of (48). Although there are 10 gain elements in $\mathbf{H}$, only four gain ones are needed to calculate it, as given in (47).

In (42) and (47), the controller gains, $k_{1}, k_{2}, k_{3}$ and $k_{4}$, are already determined from LQRq. It should be noticed that LQRq has the LQ objective function of (10). Instead of using LQRq to derive $\mathbf{H}$, the four controller gains in $\mathbf{H}$ can be directly determined by the optimization method as presented in the previous work [26].

Let the controller gain matrix be defined as $\mathbf{S}_{q}$ as (49). With $\mathbf{S}_{q}$, the full-state feedback gain matrix $\mathbf{S}_{f}$ for the FC model is obtained as (50). As given in (50), the gain matrix $\mathbf{S}_{f}$ has the form identical to (47). In other words, the full-state feedback controller for the FC model can be designed with four gain elements in $\mathbf{S}_{q}$. The problem of determining the controller gains of $\mathbf{S}_{q}$ can be formulated as an optimization one, as given in (51). In (51), $\operatorname{Re}[]$ is the function that returns real parts of complex numbers. It should be noticed that the LQ objective function of (36) or the weighting matrices of (39) is adopted for this optimization problem, (51). This problem is non-convex and nonlinear. So, it is hard to apply a convex optimization method. To solve this problem, the heuristic optimization method, i.e., the derandomized evolution strategy with covariance matrix adaptation (CMA-ES), is adopted [27]. With the optimum gains obtained by solving (51), the full-state feedback gain matrix $\mathbf{S}_{f}$ is obtained as (50). Let denote this controller $\mathbf{S}_{f}$ as LQSCfq. It is 
expected that LQSCfq is equivalent to LQRf2 and LQRfq because these have the identical LQ objective function, (36).

$$
\begin{gathered}
\mathbf{S}_{q}=\left[\begin{array}{llll}
q_{1} & q_{2} & q_{3} & q_{4}
\end{array}\right] \\
\mathbf{u}_{f}=\left(q_{1} \mathbf{G}^{T} \mathbf{C}_{1}+q_{2} \mathbf{C}_{3}+q_{3} \mathbf{G}^{T} \mathbf{C}_{2}+q_{4} \mathbf{C}_{4}\right) \mathbf{x}_{f}=\mathbf{S}_{f} \mathbf{x}_{f}
\end{gathered}
$$

$$
\begin{array}{ll}
\min _{q_{1}, q_{2}, q_{3}, q_{4}} & J_{q s}=\frac{1}{2} \operatorname{trace}\left(\mathbf{P}_{f}\right), \mathbf{P}_{f}=\mathbf{P}_{f}^{T}>0 \\
\text { s.t. } \quad & \left\{\begin{array}{l}
\max \left(\operatorname{Re}\left[\mathbf{A}_{f}+\mathbf{B}_{2 f} \mathbf{S}_{f}\right]\right)<0 \\
\left(\mathbf{A}_{f}+\mathbf{B}_{2 f} \mathbf{S}_{f}\right)^{T} \mathbf{P}_{f}+\mathbf{P}_{f}\left(\mathbf{A}_{f}+\mathbf{B}_{2 f} \mathbf{S}_{f}\right)+\mathbf{Q}_{f q}+\mathbf{S}_{f}^{T} \mathbf{N}_{f q}^{T}+\mathbf{N}_{f q} \mathbf{S}_{f}+\mathbf{S}_{f}^{T} \mathbf{R}_{f q} \mathbf{S}_{f}=0
\end{array}\right.
\end{array}
$$

\subsection{Static Output Feedback Control with Quarter-Car Model}

When using LQR for active suspension control, it is too hard to measure the state variables. For example, the state variables in the QC model, i.e., the vertical displacements and velocities of the sprung and unsprung masses are hard to measure. To cope with this problem, SOF is adopted. SOF uses available sensor signals for feedback control. For the QC model, the typical sensor signals are the suspension stroke and its rate.

The SOF controller has the form of (52). With the definition of the state vector, (7), the vector of sensor outputs, $\mathbf{y}_{q}$, in the QC model are defined as (53). As shown in (53), the sensor outputs are the suspension stroke and its rate. Therefore, there are two elements in $\mathbf{K}_{\text {SOF }}$ while $\mathbf{K}_{q}$ has four elements. By replacing $\mathbf{y}_{q}$ in (52) with the output definition of (53), the control input $u$ is obtained as (54). From (54), the gain matrix of the full-state feedback from LQ SOF controller for the QC model is given in (55). Let this controller be denoted as LQSOFq.

$$
\begin{gathered}
u=\mathbf{K}_{S O F} \mathbf{y}_{q} \\
\mathbf{y}_{q}=\left[\begin{array}{c}
z_{s}-z_{u} \\
\dot{z}_{s}-\dot{z}_{u}
\end{array}\right]=\left[\begin{array}{cccc}
1 & -1 & 0 & 0 \\
0 & 0 & 1 & -1
\end{array}\right] \mathbf{x}_{q}=\mathbf{C}_{s} \mathbf{x}_{q} \\
u=\mathbf{K}_{S O F} \mathbf{y}_{q}=\mathbf{K}_{S O F} \mathbf{C}_{s} \mathbf{x}_{q} \\
\mathbf{V}_{q} \triangleq \mathbf{K}_{S O F} \mathbf{C}_{s}
\end{gathered}
$$

LQ SOF control is to find $\mathbf{K}_{S O F}$ which gives a minimum of LQ objective function. So, this is formulated as an optimization problem, as given in (56). To find the optimum gain $\mathbf{K}_{S O F}$, the heuristic optimization method, CMA-ES, is applied [27]. After finding the gain matrix $\mathbf{V}_{q}$ or $\mathbf{K}_{S O F}$, the active suspension controller for the FC model can be obtained as (57). In (57), $v_{1}, v_{2}, v_{3}$ and $v_{4}$ are the elements of $\mathbf{V}_{q}$. Let denote this controller as LQSOFfq.

$$
\begin{aligned}
\min _{\text {SOF }} & J_{f s}=\frac{1}{2} \operatorname{trace}\left(\mathbf{P}_{s}\right), \mathbf{P}_{s}=\mathbf{P}_{s}^{T}>0 \\
\text { s.t. } & \left\{\begin{array}{r}
\max \left(\operatorname{Re}\left[\mathbf{A}_{q}+\mathbf{B}_{2 q} \mathbf{V}_{q}\right]\right)<0 \\
\left(\mathbf{A}_{q}+\mathbf{B}_{2 q} \mathbf{V}_{q}\right)^{T} \mathbf{P}_{s}+\mathbf{P}_{s}\left(\mathbf{A}_{q}+\mathbf{B}_{2 q} \mathbf{V}_{q}\right)+\mathbf{Q}_{q}+\mathbf{V}_{q}^{T} \mathbf{N}_{q}^{T}+\mathbf{N}_{q} \mathbf{V}_{q}+\mathbf{V}_{q}^{T} \mathbf{R}_{q} \mathbf{V}_{q}=0 \\
\mathbf{u}_{f}=\left(v_{1} \mathbf{G}^{T} \mathbf{C}_{1}+v_{2} \mathbf{C}_{3}+v_{3} \mathbf{G}^{T} \mathbf{C}_{2}+v_{4} \mathbf{C}_{4}\right) \mathbf{x}_{f}=\mathbf{W} \mathbf{x}_{f}
\end{array}\right.
\end{aligned}
$$

Equation (57) has the identical form to (47) and (50). In other words, LQRfq, LQSCfq, and LQSOFfq have the identical form. The difference between these controllers is how to obtain gain elements. The four gain elements of LQRfq are identical to those of LQRq. The four gain elements of LQSCfq and LQSOFfq are obtained from the optimization problem (51) and (56), respectively. The difference between LQSCfq and LQSOFfq is the model used in the optimization. While LQSCfq uses the FC model and the weighting matrices of $\mathbf{Q}_{f q}$, $\mathbf{N}_{f q}$ and $\mathbf{R}_{f q}$, LQSOFfq uses the QC one with the weighting matrices $\mathbf{Q}_{q}, \mathbf{N}_{q}$ and $\mathbf{R}_{q}$. 


\subsection{Linear Quadratic Gaussian Control with Quarter-Car Model}

As mentioned earlier, it is not easy to measure some state variables in LQR. Instead of using SOF, an observer-based controller, especially LQG, has been adopted. In this section, LQG is designed with QC and FC models. In LQG, the gain of LQR for QC model is used to design the full-state feedback controller, as given in (47).

In this paper, it is assumed that the available sensor outputs are the suspension stroke and its rate in the QC model. So, the vector of available sensor outputs is given in (53), which is identical to that of the LQ SOF controller. The state observer or Kalman filter for the QC model has the form of (58). The covariance matrices of system uncertainty and measurement noise are given as $\mathbf{M}_{q}$ and $\mathbf{N}_{q}$, respectively. The gain matrix of the observer, $\mathbf{L}_{q}$, is calculated by solving the filter algebraic Riccati equation (FARE) with the matrices $\mathbf{A}_{q}, \mathbf{C}_{s}, \mathbf{M}_{q}$ and $\mathbf{N}_{q}$. With the estimated state and the controller gain $\mathbf{K}_{q}$, the control input for the QC model is obtained as (59). The state-space equation of the controlled system and the state observer for the QC model is given as (60). This is called LQG or compensator [28]. Let this controller (60) be denoted as LQGq.

$$
\begin{gathered}
\dot{\hat{\mathbf{x}}}_{q}=\mathbf{A}_{q} \hat{\mathbf{x}}_{q}+\mathbf{B}_{2 q} u+\mathbf{B}_{1 q} z_{r}+\mathbf{L}_{q}\left(\mathbf{y}_{q}-\mathbf{C}_{s} \hat{\mathbf{x}}_{q}\right) \\
u=-\mathbf{K}_{q} \hat{\mathbf{x}}_{q} \\
{\left[\begin{array}{c}
\dot{\mathbf{x}}_{q} \\
\dot{\hat{\mathbf{x}}}_{q}
\end{array}\right]=\left[\begin{array}{cc}
\mathbf{A}_{q} & -\mathbf{B}_{2 q} \mathbf{K}_{q} \\
\mathbf{L}_{q} \mathbf{C}_{s} & \mathbf{A}_{q}-\mathbf{B}_{2 q} \mathbf{K}_{q}-\mathbf{L}_{q} \mathbf{C}_{s}
\end{array}\right]\left[\begin{array}{c}
\mathbf{x}_{q} \\
\hat{\mathbf{x}}_{q}
\end{array}\right]}
\end{gathered}
$$

For the FC model, the vector of available sensor outputs, i.e., the suspension stroke and its rate, given in (53) is represented as (61). In (61), $\mathbf{A}_{f, i}$ represents the $i$-th row of the matrix $\mathbf{A}_{f}$. The state observer or Kalman filter for the FC model has the form of (62). The covariance matrices of system uncertainty and measurement noise are given as $\mathbf{M}_{f}$ and $\mathbf{N}_{f}$, respectively. The gain matrix of the observer, $\mathbf{L}_{f}$, is calculated by solving FARE with the matrices $\mathbf{A}_{f}, \mathbf{C}_{e}, \mathbf{M}_{f}$ and $\mathbf{N}_{f}$. With the estimated state and the controller gain $\mathbf{K}_{f}$, the control input for the FC model is obtained as (63). The state-space equation with the controlled system and the observer for the FC model is given as (64). This is LQG for the FC model.

Let the LQG with the LQ objective functions of (35) and (40), i.e., LQRf1 and LQRf2, be denoted as LQGf1 and LQGf2, respectively. If the controller gain $\mathbf{K}_{f}$ is replaced with $\mathbf{H}$ in (64), the controller gain $\mathbf{K}_{q}$ can be used for LQG with FC model, as given in (65). Let the LQG of (65) be denoted as LQGfq. If the controller gain $\mathbf{H}$ is replaced with $\mathbf{S}_{f}$ in (65), the controller gain $\mathbf{S}_{q}$ can be used for LQG with FC model. Let this LQG be denoted as LQGSCfq. It is expected that LQGf2 is equivalent to LQGfq and LQGSCfq because these have the identical LQ objective function and the identical sensor signals.

$$
\begin{gathered}
\mathbf{y}_{f}=\left[\begin{array}{c}
\mathbf{z}_{s}-\mathbf{z}_{u} \\
\dot{\mathbf{z}}_{s}-\dot{\mathbf{z}}_{u}
\end{array}\right]=\left[\begin{array}{c}
\mathbf{G}^{T} \mathbf{p}-\mathbf{z}_{u} \\
\mathbf{G}^{T} \dot{\mathbf{p}}-\dot{\mathbf{z}}_{u}
\end{array}\right]=\left[\begin{array}{ccc}
\mathbf{G}^{T} & -\mathbf{I}_{4 \times 4} & 0_{4 \times 7} \\
0_{4 \times 7} & \mathbf{G}^{T} & -\mathbf{I}_{4 \times 4}
\end{array}\right] \mathbf{x}_{f}=\mathbf{C}_{e} \mathbf{x}_{f} \\
\dot{\hat{\mathbf{x}}}_{f}=\mathbf{A}_{f} \hat{\mathbf{x}}_{f}+\mathbf{B}_{2 f} \mathbf{u}_{f}+\mathbf{B}_{1 f} \mathbf{w}_{f}+\mathbf{L}_{f}\left(\mathbf{y}_{f}-\mathbf{C}_{e} \hat{\mathbf{x}}_{f}\right) \\
\mathbf{u}_{f}=-\mathbf{K}_{f} \hat{\mathbf{x}}_{f} \\
{\left[\begin{array}{c}
\dot{\mathbf{x}}_{f} \\
\dot{\hat{\mathbf{x}}}_{f}
\end{array}\right]=\left[\begin{array}{cc}
\mathbf{A}_{f} & -\mathbf{B}_{2 f} \mathbf{K}_{f} \\
\mathbf{L}_{f} \mathbf{C}_{e} & \mathbf{A}_{f}-\mathbf{B}_{2 f} \mathbf{K}_{f}-\mathbf{L}_{f} \mathbf{C}_{e}
\end{array}\right]\left[\begin{array}{c}
\mathbf{x}_{f} \\
\hat{\mathbf{x}}_{f}
\end{array}\right]} \\
{\left[\begin{array}{c}
\dot{\mathbf{x}}_{f} \\
\dot{\mathbf{x}}_{f}
\end{array}\right]=\left[\begin{array}{cc}
\mathbf{A}_{f} & \mathbf{B}_{2 f} \mathbf{H} \\
\mathbf{L}_{f} \mathbf{C}_{e} & \mathbf{A}_{f}+\mathbf{B}_{2 f} \mathbf{H}-\mathbf{L}_{f} \mathbf{C}_{e}
\end{array}\right]\left[\begin{array}{c}
\mathbf{x}_{f} \\
\hat{\mathbf{x}}_{f}
\end{array}\right]}
\end{gathered}
$$

\section{Frequency-Domain Analysis on Designed Controllers}

In this section, frequency-domain analysis is done to check the performance of the designed controllers. Through frequency-domain analysis, full-state feedback, static output 
feedback and observer-based output feedback controllers, i.e., LQR, LQSOF, and LQG, are compared to one another.

Table 1 shows the parameters of 2-DOF QC and 7-DOF FC models, which were referred from E-Class sedan given in CarSim [29]. Weights in LQ objective functions, (10), (32), and (36), are given in Table 2. Those weights emphasize the ride comfort, i.e., the reduction of the vertical acceleration of the sprung mass. As a consequence, the road adhesion will deteriorate. For this reason, frequency responses within the range from 4 to $10 \mathrm{~Hz}$ should be focused, as given in Figure 1. In case of the roll and pitch angles, frequency responses within the range from 0.5 to $2 \mathrm{~Hz}$ should be focused. In this paper, it is assumed that the actuator used to generate the control input has infinite bandwidth. In other words, delay and saturation of an actuator are not taken into account. The covariance matrices of system uncertainty and measurement noise used in designing LQG are given in Table 3.

Table 1. Parameters of the 7-DOF full-car model [29].

\begin{tabular}{cccc}
\hline$m_{s}$ & $1653.0 \mathrm{~kg}$ & $m_{u}$ & $45.0 \mathrm{~kg}$ \\
$I_{x}$ & $614.0 \mathrm{~kg} \cdot \mathrm{m}^{2}$ & $I_{y}$ & $2765.0 \mathrm{~kg} \cdot \mathrm{m}^{2}$ \\
$l_{f}$ & $1.402 \mathrm{~m}$ & $l_{r}$ & $1.646 \mathrm{~m}$ \\
$t_{f}, t_{r}$ & $0.8 \mathrm{~m}$ & $k_{t}$ & $230,000 \mathrm{~N} / \mathrm{m}$ \\
$k_{s}$ & $34,000 \mathrm{~N} / \mathrm{m}$ & $b_{s}$ & $3500 \mathrm{~N} \cdot \mathrm{s} / \mathrm{m}$ \\
\hline
\end{tabular}

Table 2. Maximum allowable values in LQ cost function.

\begin{tabular}{cccccc}
\hline$\xi_{1}$ & $0.2 \mathrm{~m} / \mathrm{s}^{2}$ & $\xi_{2}$ & $10 \mathrm{deg} / \mathrm{s}^{2}$ & $\xi_{3}$ & $10 \mathrm{deg} / \mathrm{s}^{2}$ \\
$\xi_{4}$ & $3 \mathrm{deg}$ & $\xi_{5}$ & $10 \mathrm{deg} / \mathrm{s}$ & $\xi_{6}$ & $3 \mathrm{deg}$ \\
$\xi_{7}$ & $10 \mathrm{deg} / \mathrm{s}$ & $\xi_{8}$ & $0.2 \mathrm{~m}$ & $\xi_{9}$ & $0.2 \mathrm{~m}$ \\
$\xi_{10}$ & $3000 \mathrm{~N}$ & $\eta_{1}, \chi_{1}$ & $\xi_{1}$ & $\eta_{2}, \chi_{2}$ & $\xi_{8}$ \\
$\eta_{3}, \chi_{3}$ & $\xi_{9}$ & $\eta_{4}, \chi_{4}$ & $\xi_{10}$ & & \\
\hline
\end{tabular}

Table 3. Covariance matrices in LQG for quarter-car and full-car models.

\begin{tabular}{cccc}
\hline $\mathbf{M}_{q}$ & $10^{4} \cdot \mathbf{I}_{4 \times 4}$ & $\mathbf{N}_{q}$ & $10^{-4}$ \\
$\mathbf{M}_{f}$ & $10^{4} \cdot \mathbf{I}_{14 \times 14}$ & $\mathbf{N}_{f}$ & $10^{-4} \cdot \mathbf{I}_{8 \times 8}$ \\
\hline
\end{tabular}

\subsection{Full-State Feedback Case}

The first type of frequency-domain analysis is done for the full-state feedback controllers. For frequency-domain analysis, the FC model is used. Figure 5 shows the frequency responses of each output from the road profile $z_{r 1}$ with the FC model for each controller. The gain matrices of $\mathbf{K}_{q}$ and $\mathbf{S}_{q}$ of LQRq and LQSCfq are given in Table 4. LQRq was obtained by solving Riccati equation with the state-space Equation (9), the LQ objective function (10) and Table 2. LQSCfq was obtained by solving (51) with the state-space Equation (31), LQ objective function (36) and Table 2. As expected in the Section 2.3, LQRfq and LQSCfq has nearly identical elements as given in Table 4.

As shown in Figure 5a, LQRf2 is better than LQRf1 in terms of the ride comfort. This means that higher emphasis on the vertical acceleration at each corner in (36) is better than higher emphasis on the vertical, roll and pitch accelerations in (32). On the other hand, LQRf1 is better than LQRf1 in terms of the roll and pitch angles. This is caused by the fact that the LQ objective function of LQRf2, (36), has no terms on the roll and pitch angles.

As shown in Figure 5, LQRf2, LQRfq and LQSCfq show nearly identical performances in terms of the ride comfort and the road adhesion. The measures of the ride comfort, i.e., the vertical acceleration between 4 and $10 \mathrm{~Hz}$, the roll and pitch angle between 0.5 and $2 \mathrm{~Hz}$ are improved by the controllers, as shown in Figure $5 \mathrm{a}-\mathrm{c}$, respectively. This is natural because these were designed with the identical LQ objective functions. From these results, it can be concluded that the controllers derived from LQRq, i.e., LQRfq and LQSCfq, can give equivalent performance to that designed with the FC one, LQRf2. This is quite important that a much smaller number of gains are needed in designing the active 
suspension controller for the FC model. In other words, only four gain elements of LQRq can give nearly identical performance to LQRf2 which needs 56 gain elements in the active suspension controller for the FC model.

$$
z_{r 1} \rightarrow \ddot{z}_{c}
$$

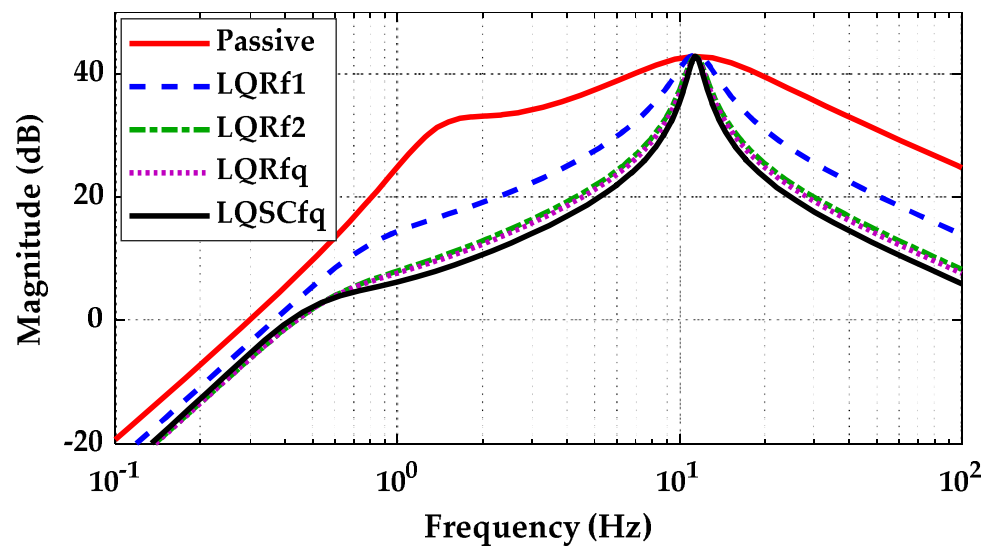

(a)

$$
z_{r 1} \rightarrow \phi
$$

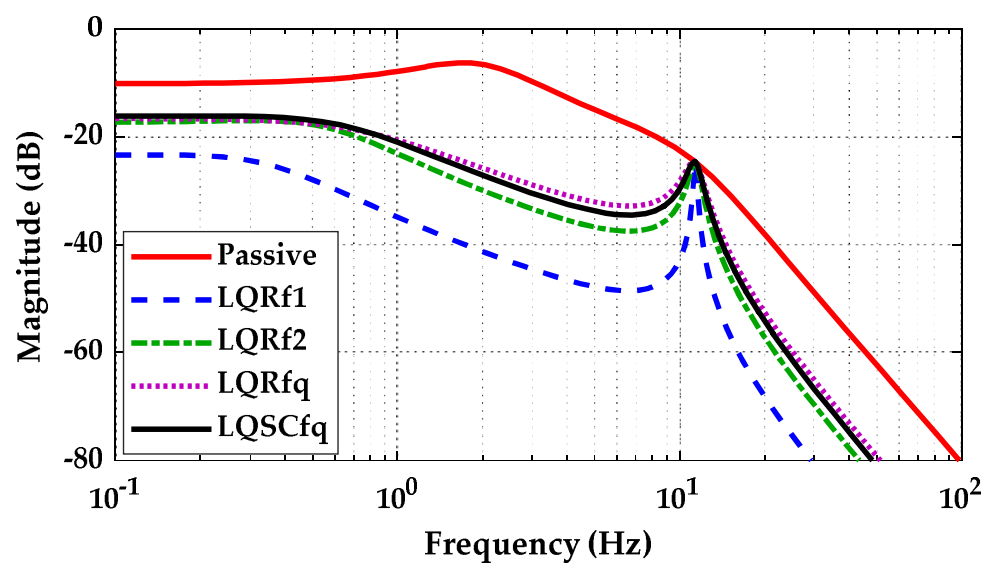

(b)

$$
z_{r 1} \rightarrow \theta
$$

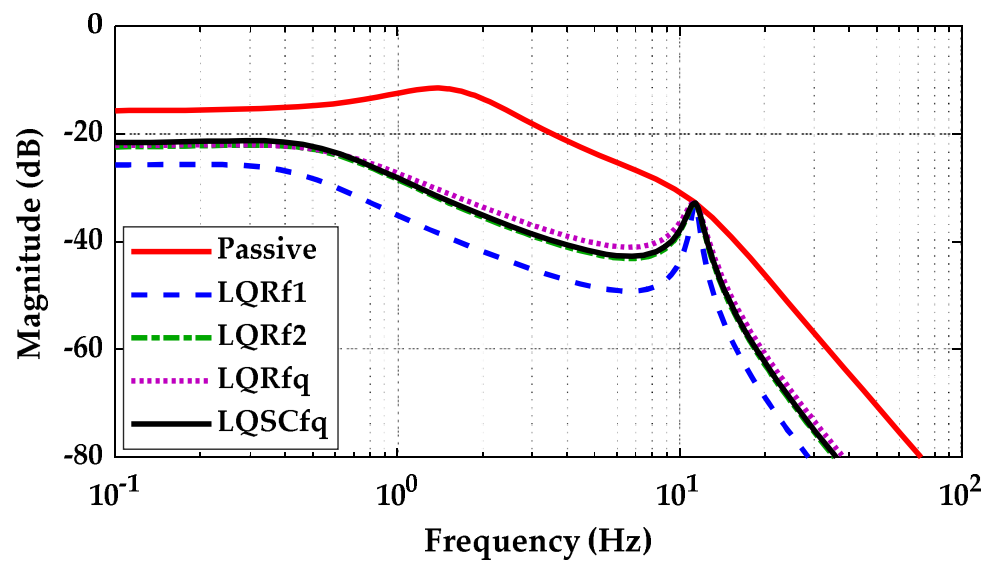

(c)

Figure 5. Cont. 


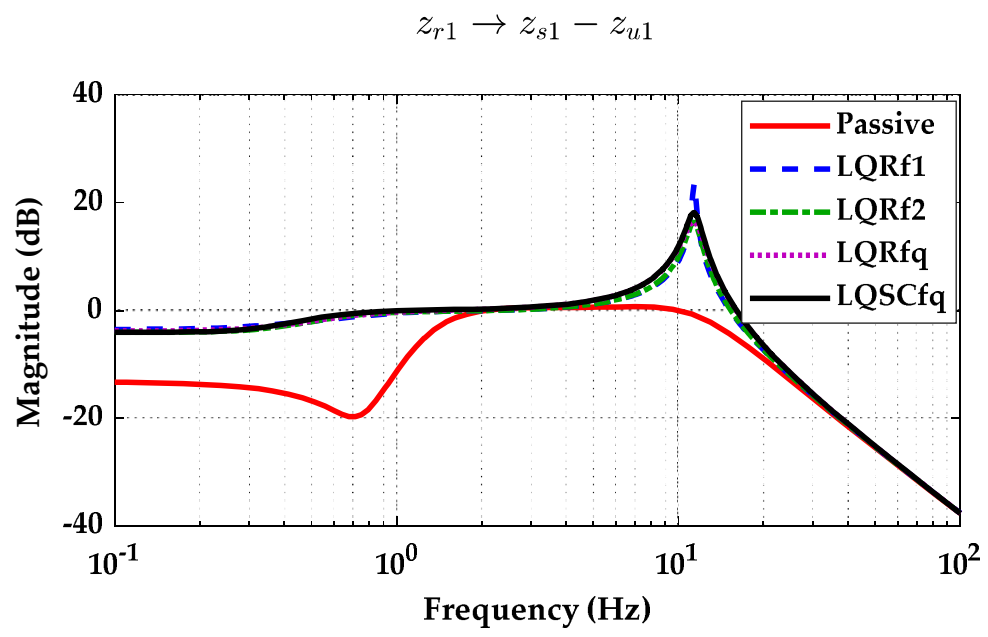

(d)

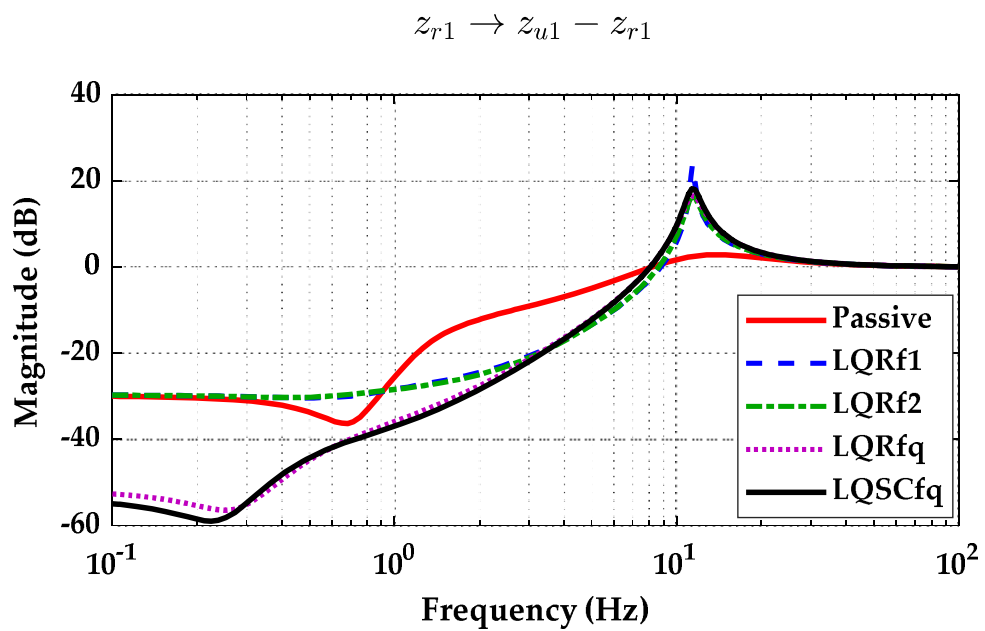

(e)

Figure 5. Frequency responses from the road profile $z_{r 1}$ to each output for full-state feedback case. (a) Vertical acceleration of the sprung mass; (b) Roll angle of the sprung mass; (c) Pitch angle of the sprung mass; (d) Suspension stroke; (e) Tire deflection.

Table 4. Gain matrices of LQR and LQSq.

\begin{tabular}{|c|c|c|c|c|}
\hline $\mathbf{K}_{q}$ & $-28,929.0$ & $31,583.0$ & -1538.4 & 3017.5 \\
\hline $\mathbf{S}_{q}$ & $-30,152.0$ & $32,070.0$ & -1968.9 & 3102.7 \\
\hline
\end{tabular}

Another feature of the controllers is that the suspension strokes of the controlled suspensions are nearly identical to one another within the frequency range from 0.1 to $6 \mathrm{~Hz}$, and larger than the passive suspension as shown in Figure 5d. Moreover, the tire deflections of these controllers were smaller than that of the passive system, as shown in Figure 5e. This means that the active suspension controllers enhance the ride comfort by generating larger motion of the suspension stroke, and that the road adhesion was deteriorated by those controllers as a result. This is a natural consequence of the active suspension control. Hereafter, the frequency responses of the suspension stroke and the tire deflection are not presented because it will show identical tendency regardless of controllers.

Table 5 shows the decibel scale magnitudes of frequency responses for each controller. The values in Table 5 are calculated from the frequency responses in Figure 5. The magnitudes of the vertical acceleration, the suspension stroke and the tire deflection are sampled at $6 \mathrm{~Hz}$, which is the peak frequency of Wk in Figure 1. The magnitudes of the roll and 
pitch angles are sampled at $1 \mathrm{~Hz}$, which is the peak frequency of Wd in Figure 1. As shown Table 5, the LQRf2, LQRfq and LQSCfq show nearly similar performance to one another. Moreover, the ride comfort enhancement achieved by these controllers are better than LQRf1. For example, LQRfq reduced the vertical acceleration from $38.9 \mathrm{~dB}$ and $29.9 \mathrm{~dB}$ the passive system and LQRf1 to $23.7 \mathrm{~dB}$, respectively. These results mean that the vertical acceleration of the passive system and LQRf1 were reduced by $39 \%$ and $20 \%$ by virtue of LQRfq, respectively. Among these controllers, LQSCfq shows the best performance in terms of the vertical acceleration. On the other hand, the roll and pitch angles of LQRf1 are better than the three controllers because the LQ objective function of LQRf1 has the terms emphasizing the roll and pitch angles. As shown in Figure 5 and Table 5, the suspension stroke increases, and the tire deflection decreases as the vertical acceleration is reduced by the controllers. The smaller the tire deflection, the smaller the vertical and longitudinal/lateral tire forces. As a result, the road adhesion is deteriorated.

Table 5. Magnitudes of frequency responses for each controller (dB scale).

\begin{tabular}{cccccc}
\hline & $\ddot{z}_{c}$ & $\boldsymbol{\phi}$ & $\boldsymbol{\theta}$ & $z_{\boldsymbol{s} \mathbf{1}}-z_{\boldsymbol{u} \mathbf{1}}$ & $\boldsymbol{z}_{\boldsymbol{u} \mathbf{1}}-z_{\boldsymbol{r} \mathbf{1}}$ \\
\hline Passive & 38.9 & -7.8 & -12.4 & 0.6 & -3.1 \\
LQRf1 & 29.9 & -34.9 & -35.1 & 1.9 & -9.8 \\
LQRf2 & 24.4 & -23.1 & -28.5 & 1.9 & -9.8 \\
LQRfq & 23.7 & -20.6 & -27.3 & 2.8 & -8.0 \\
LQSCfq & 22.0 & -21.0 & -28.2 & 2.8 & -8.2 \\
\hline
\end{tabular}

\subsection{Static Output Feedback Case}

The second type of frequency-domain analysis is done for the LQ SOF controllers. Figure 6 shows the frequency responses of each output from the road profile $z_{r 1}$ with the FC model for each controller. The gain matrices of $\mathbf{K}_{S O F}$ and $\mathbf{V}_{q}$ of LQSOFq are given in Table 6 . The gain $\mathbf{K}_{S O F}$ of LQSOF is obtained by solving (56) with the LQ objective function (10) and Table 2.

As shown in Figure 6, all the measures of LQSOFfq designed with the QC model are nearly identical to LQRf2 and LQRfq within the frequency range from 2 to $10 \mathrm{~Hz}$. This means that only two gain elements of $\mathbf{K}_{S O F}$ can give the equivalent performance to LQRf2 and LQRfq, the active suspension controller designed with the FC model. The first reason why LQSOFfq gives good performance equivalent to LQRf2 and LQRfq is that the controller structure of LQSOFfq is identical to the suspension force (1). In other words, the first and second gain elements of $\mathbf{K}_{S O F}$ correspond to $k_{S}$ and $b_{S}$ of (1), respectively. If those of $\mathbf{K}_{S O F}$ coincide to $k_{s}$ and $b_{s}$ of (1), the forces generated from tire cannot be transmitted to the sprung mass. The second reason is that these controllers have identical LQ objective functions of (10) and (36).

As shown in Figure 6, the drawback of LQSOFfq is that its performance is deteriorated in the frequency range below $1 \mathrm{~Hz}$. As shown in Figure 1, the motion sickness near $0.2 \mathrm{~Hz}$ and the horizontal motion near $1 \mathrm{~Hz}$ are important in terms of the ride comfort. The vertical acceleration of the sprung mass of LQSOFfq is worse than the passive system below $0.5 \mathrm{~Hz}$, as shown in Figure 6a. Moreover, the roll and pitch angles of the sprung mass of LQSOFfq are worse than the passive system below $0.7 \mathrm{~Hz}$, as shown in Figure 6b,c. This is critical because it deteriorates the motion sickness and the ride comfort along the horizontal direction. For this reason, LQSOFfq is not recommended for the ride comfort in spite of its good performance along the vertical direction and the simplicity in controller design procedure.

\subsection{LQG Case}

The third type of frequency-domain analysis is done for the observer-based controllers, i.e., LQG. Figure 7 shows the frequency responses of each output from the road profile $z_{r}$ with the QC model for LQRq, LQSOFq and LQGq, as given in (60). 
As shown in Figure 7a, the performance of LQGq is slightly worse than that of LQRq and LQSOFq. Moreover, it becomes worse than LQRq and LQSOFq below $4 \mathrm{~Hz}$ and above $40 \mathrm{~Hz}$ in terms of the ride comfort. This tendency of LQGq is nearly same as that of LQSOFq, as given in Figure 6. This is caused by the fact that LQSOFq and LQGq use identical sensor signals. Nevertheless, the performance of LQGq is satisfactory in terms of the ride comfort because it shows nearly identical performance to $L Q R q$ within the frequency range from 4 to $10 \mathrm{~Hz}$. Moreover, the performance of LQGq is better than LQSOFq for the frequency range below $0.6 \mathrm{~Hz}$, as shown in Figure 7a. The frequency responses of the suspension stroke and the tire deflection in Figure $7 \mathrm{~b}, \mathrm{c}$ show nearly identical tendency to those of Figure 5d,e, respectively.

These results are varied according to the selection of the covariance matrices, i.e., $\mathbf{M}_{q}$ and $\mathbf{N}_{q}$ under the condition that an identical set of weights in LQ objective function is used. It also depends on the selection of the available sensor outputs, as given in (53). If LQSOFq and LQGq use identical sensor signals for output feedback, these can give nearly identical performances, as shown in Figure 7. From these results, it can be concluded that LQG can be adopted as an output feedback controller for the purpose of enhancing the ride comfort.

Figure 8 shows the frequency responses of each output from the road profile $z_{r 1}$ with the FC model for each controller. As shown in Figure 8, the relationship between LQRq and LQGq given in Figure 7 is nearly identical to that of LQRfq and LQGfq. In other words, the performance of LQGfq becomes worse than LQRfq below $4 \mathrm{~Hz}$ and above $40 \mathrm{~Hz}$ in terms of the ride comfort. This can be checked in Figures 7a and 8a. Moreover, the ride comfort of LQGq becomes identical to the passive system below $0.5 \mathrm{~Hz}$, as shown in Figure 7a. This is also confirmed by the frequency responses of the passive system and LQGfq in Figure 8a. Nevertheless, the LQG controllers, i.e., LQGf2, LQGfq and LQGSCfq, show good performance within the frequency range from 2 to $10 \mathrm{~Hz}$, in terms of the ride comfort.

As shown in Figure 8, the frequency responses of LQGf2, LQGfq and LQGSCfq show identical tendency as LQRf2, LQRfq and LQSCfq, as given in Figure 5. This is confirmed by the fact that the frequency responses of these controllers are nearly identical to each other. This is quite natural because these have the identical LQ objective function and the identical Kalman filter. Moreover, LQGfq and LQGSCfq have the identical controller structure and nearly identical controller gains, as given in Table 4.

Figure 7 did not show the frequency responses of the roll and pitch angles because the QC model has no roll and pitch motions. As shown in Figure 8b,c, the LQG controllers show identical performance to the passive system below $0.6 \mathrm{~Hz}$. This means that the LQG controllers, i.e., LQGf2, LQGfq and LQGSCfq, are not effective in controlling the roll and pitch motions for the ride comfort along the horizontal direction and the motion sickness.

$$
z_{r 1} \rightarrow \ddot{z}_{c}
$$

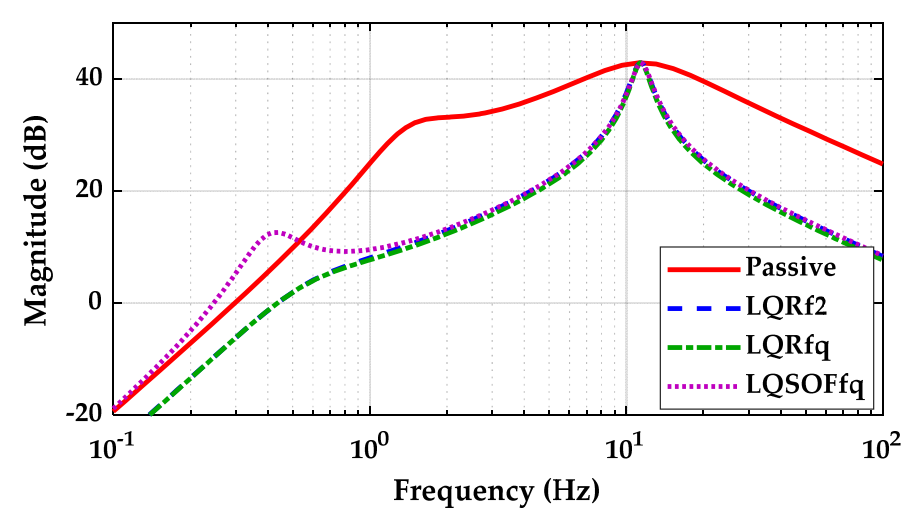

(a)

Figure 6. Cont. 


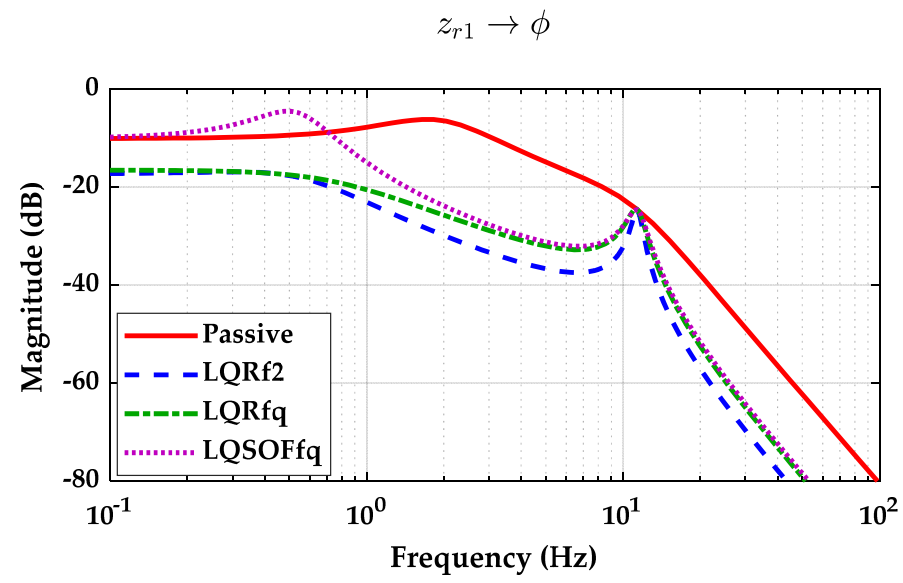

(b)

$z_{r 1} \rightarrow \theta$

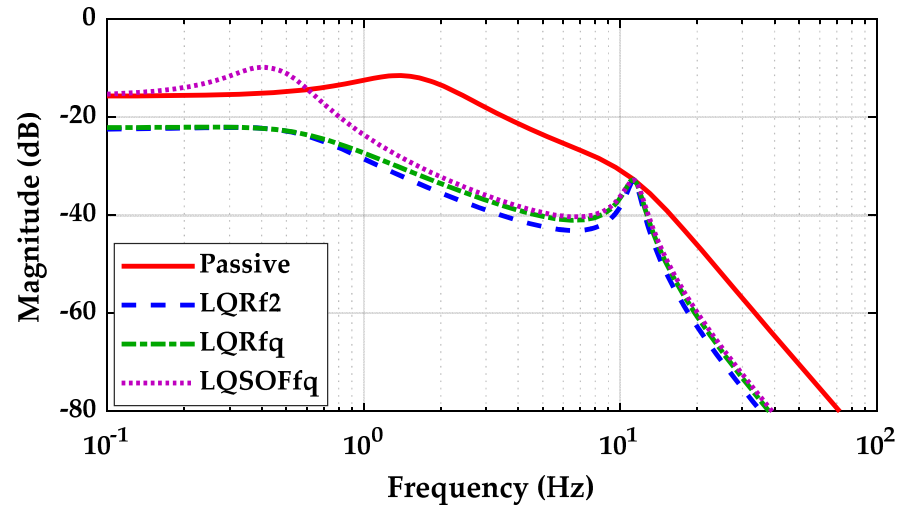

(c)

Figure 6. Frequency responses from the road profile $z_{r 1}$ to each output for static output feedback case. (a) Vertical acceleration of the sprung mass; (b) Roll angle of the sprung mass; (c) Pitch angle of the sprung mass.

Table 6. Gain matrices of LQSOF and LQSOFq.

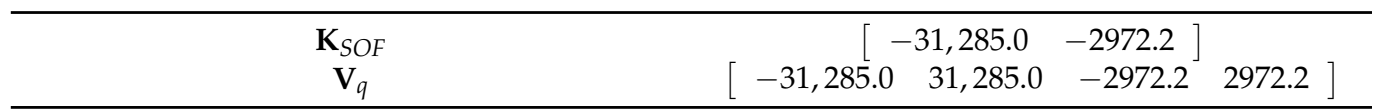

$z_{r} \rightarrow \ddot{z}_{c}$

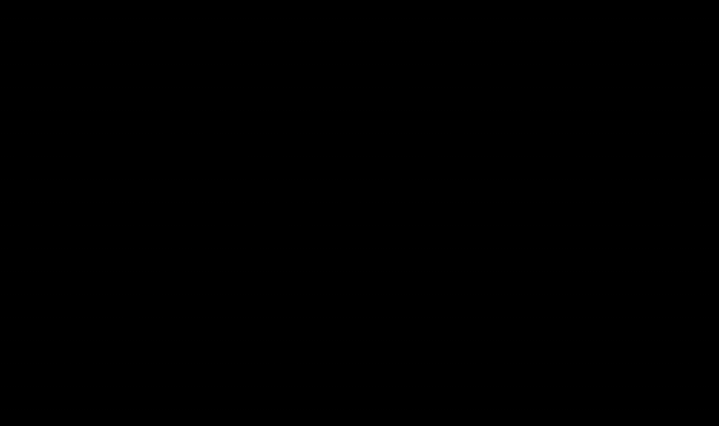

(a)

Figure 7. Cont. 
$z_{r} \rightarrow z_{s}-z_{u}$

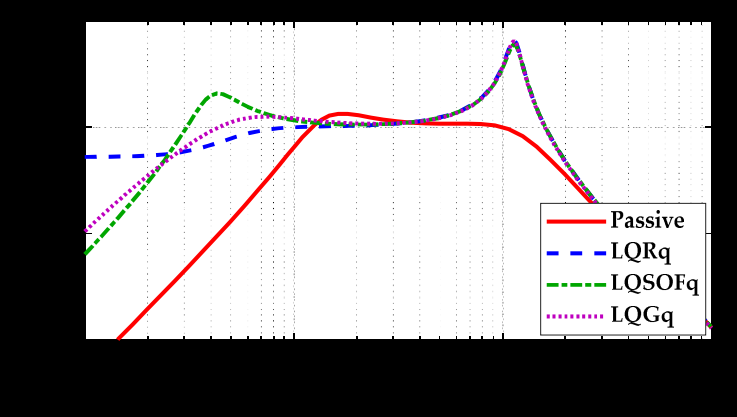

(b)

$z_{r} \rightarrow z_{u}-z_{r}$

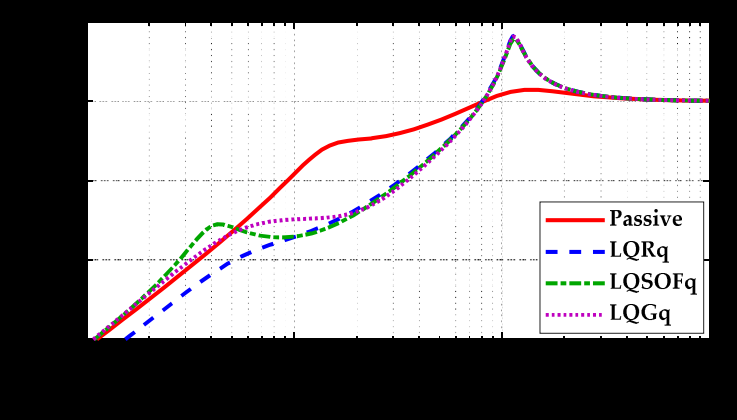

(c)

Figure 7. Frequency responses from the road profile $z_{r}$ to each output for the quarter-car model. (a) Vertical acceleration of the sprung mass; (b) Suspension stroke; (c) Tire deflection.

$z_{r 1} \rightarrow \ddot{z}_{c}$

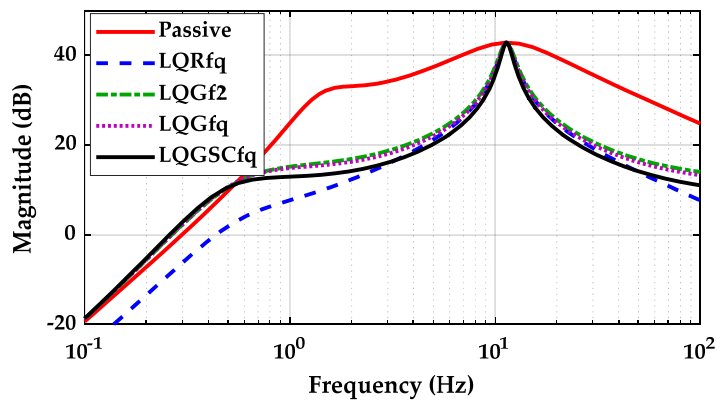

(a)

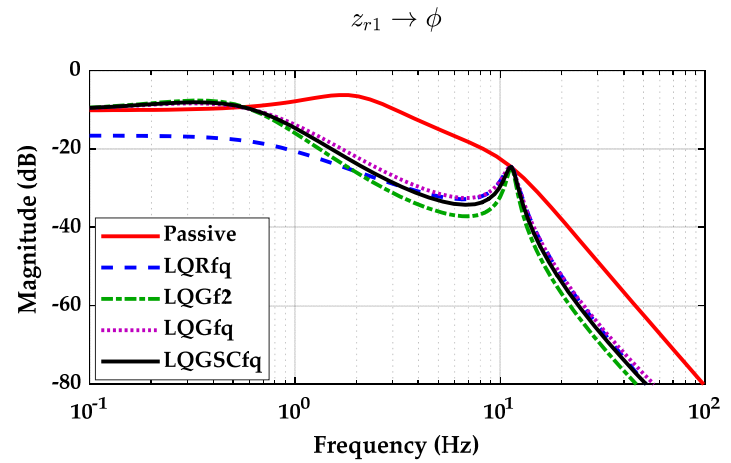

(b)

Figure 8. Cont. 


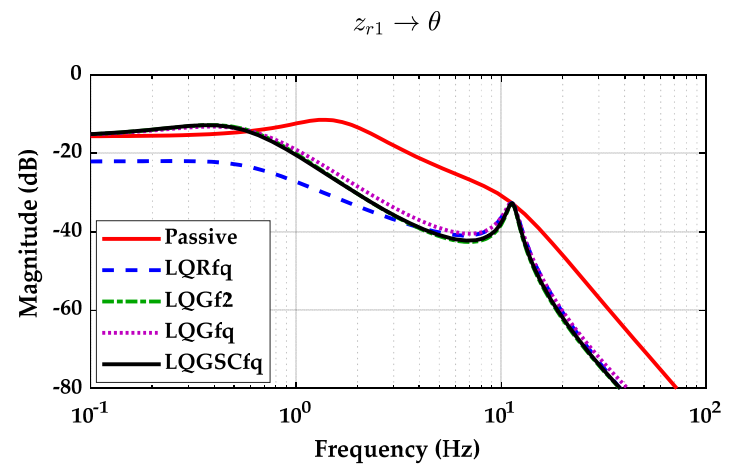

(c)

Figure 8. Frequency responses from the road profile $z_{r 1}$ to each output for LQG case. (a) Vertical acceleration of the sprung mass; (b) Roll angle of the sprung mass; (c) Pitch angle of the sprung mass.

\section{Conclusions}

In this paper, the controllers for active suspension were designed with the 2-DOF QC and 7-DOF FC models. Two LQRs, i.e., LQRq and LQRf2, were designed with the QC and FC models based on the identical LQ objective functions. To cope with the problem in measuring the state variables of the FC model, LQRq designed with the QC model was used to derive the full-state feedback controller for the FC model, i.e., LQRfq. In deriving LQRfq, only four gain elements of LQRq are needed. To cope with the identical problem, SOF and LQG controllers, i.e., LQSOFq and LQGq, are designed with the QC models and used to derive the controllers for the FC model, i.e., LQSOFfq and LQGfq. In deriving LQSOFfq, only two gain elements of LQSOFq are needed. To verify the fact that the full-state feedback controllers, i.e., LQRfq and LQGfq, derived from LQRq can give equivalent or better performance than LQRf2 and LQGf2 designed with the FC model in terms of the ride comfort, frequency-domain analysis was conducted for full-state feedback, static output feedback and observer-based output feedback controllers. From the analysis, it can be concluded that the controllers with much simpler structure or much smaller gain elements designed with the QC model, i.e., LQRfq, LQSOFfq and LQGfq, can give equivalent performance to LQRf2. The vertical acceleration of the passive system and LQRf1 were reduced by $39 \%$ and $20 \%$ by virtue of LQRfq, respectively. Further research can include comparison between robust control methodologies in designing active suspension controllers with the QC model for the FC one. Moreover, it can also include test results on a vehicle simulation package and experimental validation on real vehicles.

Author Contributions: Conceptualization, M.P. and S.Y.; methodology, S.Y.; software, S.Y.; validation, M.P. and S.Y.; formal analysis, S.Y.; investigation, M.P.; resources, M.P.; data curation, M.P.; writingoriginal draft preparation, S.Y.; writing-review and editing, M.P.; visualization, S.Y.; supervision, M.P.; project administration, M.P.; funding acquisition, S.Y. All authors have read and agreed to the published version of the manuscript.

Funding: This research received no external funding.

Institutional Review Board Statement: Not applicable.

Informed Consent Statement: Not applicable.

Data Availability Statement: All the experimental data are generated during the study at a local computer. Therefore, it cannot be made publicly available for other researchers.

Acknowledgments: This study was supported by the Research Program funded by the Seoul National University of Science and Technology.

Conflicts of Interest: The authors declare no conflict of interest. 


\section{References}

1. $\quad$ ISO 2631-1. Mechanical Vibration and Shock-Evaluation of Human Exposure to Whole-Body Vibration-Part 1: General Requirements; International Organization for Standardization: Geneva, Switzerland, 1997.

2. Rimell, A.N.; Mansfield, N.J. Design of digital filters for frequency weightings required for risk assessments of workers exposed to vibration. Ind. Health 2007, 45, 512-519. [CrossRef]

3. Tseng, H.E.; Hrovat, D. State of the art survey: Active and semi-active suspension control. Veh. Syst. Dyn. 2015, 53, 1034-1062. [CrossRef]

4. Sharp, R.S.; Crolla, D.A. Road vehicle suspension system design-A review. Veh. Syst. Dyn. 1987, 16, 167-192. [CrossRef]

5. Hrovat, D. Survey of advanced suspension developments and related optimal control applications. Automatica 1997, 33, 1781-1817. [CrossRef]

6. Cao, D.; Song, X.; Ahmadian, M. Editors' perspectives: Road vehicle suspension design, dynamics, and control. Veh. Syst. Dyn. 2011, 49, 3-28. [CrossRef]

7. Theunissen, J.; Tota, A.; Gruber, P.; Dhaens, M.; Sorniotti, A. Preview-based techniques for vehicle suspension control: A state-of-the-art review. Annu. Rev. Control 2021, 51, 206-235. [CrossRef]

8. Al-Ashmori, M.; Wang, X.A. Systematic literature review of various control techniques for active seat suspension systems. Appl. Sci. 2020, 10, 1148. [CrossRef]

9. Sun, W.; Pan, H.; Zhang, Y.; Gao, H. Multi-objective control for uncertain nonlinear active suspension systems. Mechatronics 2014, 24, 318-327. [CrossRef]

10. Chen, S.-A.; Wang, J.-C.; Yao, M.; Kim, Y.-B. Improved optimal sliding mode control for a non-linear vehicle active suspension system. J. Sound Vib. 2017, 395, 1-25. [CrossRef]

11. Wang, R.; Jing, H.; Yan, F.; Karimi, H.R.; Chen, N. Optimization and finite-frequency $H_{\infty}$ control of active suspensions in in-wheel motor driven electric ground vehicles. J. Frankl. Inst. 2015, 352, 468-484. [CrossRef]

12. Liu, M.; Gu, F.; Zhang, Y. Ride comfort optimization of in-wheel-motor electric vehicles with in-wheel vibration absorbers Energies 2017, 10, 1647. [CrossRef]

13. Jin, X.; Wang, J.; Sun, S.; Li, S.; Yang, J.; Yan, Z. Design of constrained robust controller for active suspension of in-wheel-drive electric vehicles. Mathematics 2021, 9, 249. [CrossRef]

14. Li, H.; Yu, J.; Hilton, C.; Liu, H. Adaptive sliding-mode control for nonlinear active suspension vehicle systems using T-S fuzzy approach. IEEE Trans. Ind. Electron. 2013, 60, 3328-3338. [CrossRef]

15. Yatak, M.O.; Sahin, F. Ride comfort-road holding trade-off improvement of full vehicle active suspension system by interval type-2 fuzzy control. Eng. Sci. Technol. Int. J. 2021, 24, 259-270. [CrossRef]

16. Mrazgua, J.; Chaibi, R.; Tissir, E.H.; Ouahi, M. Static output feedback stabilization of T-S fuzzy active suspension systems. J. Terramech. 2021, 97, 19-27. [CrossRef]

17. Wilson, D.A.; Sharp, R.S.; Hassan, S.A. The application of linear optimal control theory to the design of active automobile suspensions. Veh. Syst. Dyn. 1987, 15, 105-118. [CrossRef]

18. Hac, A. Optimal linear preview control of active vehicle suspension. Veh. Syst. Dyn. 1992, 21, 167-195. [CrossRef]

19. Abdel-Hady, M.B.A.; Crolla, D.A. Active suspension control algorithms for a four wheel vehicle model. Int. J. Veh. Des. 1992, 13, 144-158.

20. Mudduluru, S.R.; Chizari, M. Quarter and full car models optimisation of passive and active suspension system using genetic algorithm. arXiv 2021, arXiv:2101.12629. Available online: https:/ /arxiv.org/abs/2101.12629 (accessed on 30 August 2021).

21. Camino, J.F.; Zampieri, D.E.; Peres, P.L.D. Design of a vehicular suspension controller by static output feedback. In Proceedings of the American Control Conference, San Diego, CA, USA, 2-4 June 1999; pp. 3168-3171.

22. Elmadany, M.M.; Al-Majed, M.I. Quadratic synthesis of active controls for a quarter-car model. J. Vib. Control 2001, 7, 1237-1252. [CrossRef]

23. Lu, J.; DePoyster, M. Multiobjective optimal suspension control to achieve integrated ride and handling performance. IEEE Trans. Control Syst. Technol. 2002, 10, 807-821.

24. Jung, Y.H.; Choi, J.W.; Seo, Y.B. Overlapping decentralized EA control design for an active suspension system of a full car model. In Proceedings of the 39th SICE Annual Conference, Iizuka, Japan, 28 July 2000; pp. 85-90.

25. Bryson, A.E., Jr.; Ho, Y. Applied Optimal Control; Hemisphere: New York, NY, USA, 1975.

26. Yim, S. Design of a robust controller for rollover prevention with active suspension and differential braking. J. Mech. Sci. Technol. 2012, 26, 213-222. [CrossRef]

27. Hansen, N.; Muller, S.D.; Koumoutsakos, P. Reducing the time complexity of the derandomized evolution strategy with covariance matrix adaptation (CMA-ES). Evol. Comput. 2003, 11, 1-18. [CrossRef] [PubMed]

28. Stengel, R.F. Stochastic Optimal Control: Theory and Application; A Wiley-Interscience Publication: New York, NY, USA, 1986.

29. Mechanical Simulation Corporation. CarSim Data Manual; Version 8; Mechanical Simulation Corporation: Ann Arbor, MA, USA, 2009. 